Draft VERSION MAY 23, 2016

Preprint typeset using $\mathrm{L}^{\mathrm{A}} \mathrm{E} \mathrm{E}$ style emulateapj v. 05/12/14

\title{
CARBON AND OXYGEN ABUNDANCES IN LOW METALLICITY DWARF GALAXIES ${ }^{* \dagger}$
}

\author{
Danielle A. Berg ${ }^{1}$, Evan D. Skillman ${ }^{2}$, Richard B.C. Henry ${ }^{3}$, Dawn K. Erb ${ }^{1}$, Leticia Carigi ${ }^{4}$ \\ Draft version May 23, 2016
}

\begin{abstract}
The study of carbon and oxygen abundances yields information on the time evolution and nucleosynthetic origins of these elements, yet remains relatively unexplored. At low metallicities $(12+\log (\mathrm{O} / \mathrm{H})$ $<8.0$ ), nebular carbon measurements are limited to rest-frame UV collisionally excited emission lines. Therefore, we present UV spectrophotometry of 12 nearby, low-metallicity, high-ionization H II regions in dwarf galaxies obtained with the Cosmic Origins Spectrograph on the Hubble Space Telescope. We present the first analysis of the $\mathrm{C} / \mathrm{O}$ ratio in local galaxies based solely on simultaneous significant detections of the $\mathrm{UV} \mathrm{O}^{+2}$ and $\mathrm{C}^{+2}$ collisionally excited lines in seven of our targets and five objects from the literature, to create a final sample of 12 significant detections. Our sample is complemented by optical SDSS spectra, from which we measured the nebular physical conditions and oxygen abundances using the direct method.

At low metallicity $(12+\log (\mathrm{O} / \mathrm{H})<8.0)$, no clear trend is evident in $\mathrm{C} / \mathrm{O}$ vs. $\mathrm{O} / \mathrm{H}$ for the present sample given the large dispersion observed. When combined with recombination line observations at higher values of $\mathrm{O} / \mathrm{H}$, a general trend of increasing $\mathrm{C} / \mathrm{O}$ with increasing $\mathrm{O} / \mathrm{H}$ is also viable, but with some significant outliers. Additionally, we find the $\mathrm{C} / \mathrm{N}$ ratio appears to be constant (but with significant scatter) over a large range in oxygen abundance, indicating carbon is predominantly produced by similar nucleosynthetic mechanisms as nitrogen. If true, and our current understanding of nitrogen production is correct, this would indicate that primary production of carbon (a flat trend) dominates at low metallicity, but quasi-secondary production (an increasing trend) becomes prominent at higher metallicities. A larger sample will be needed to determine the true nature and dispersion of the relation.

Subject headings: galaxies: abundances - galaxies: evolution
\end{abstract}

\section{INTRODUCTION}

A key tracer of galaxy evolution is the change in the chemical composition over time. The metallicity of a galaxy is a sensitive observational diagnostic of its past star formation history (SFH) and present-day evolutionary state because metallicity increases monotonically with each successive generation of massive stars (MS) (Wheeler et al. 1989). Oxygen abundance is an important tracer of metallicity because it is the most abundant element in the Universe after hydrogen and helium and is convenient to observe since its emission lines are ubiquitous in the rest-frame optical regime. After oxygen, carbon and nitrogen play the largest role in the chemi-

*BASED ON OBSERVATIONS MADE WITH THE NASA/ESA HUBBLE SPACE TELESCOPE, OBTAINED FROM THE DATA ARCHIVE AT THE SPACE TELESCOPE SCIENCE INSTITUTE, WHICH IS OPERATED BY THE ASSOCIATION OF UNIVERSITIES FOR RESEARCH IN ASTRONOMY, INC., UNDER NASA CONTRACT NAS 5-26555.

†SOME OBSERVATIONS REPORTED HERE WERE OBTAINED AT THE MMT OBSERVATORY, A JOINT FACILITY OF THE UNIVERSITY OF ARIZONA AND THE SMITHSONIAN INSTITUTION.

${ }^{1}$ Center for Gravitation, Cosmology and Astrophysics, Department of Physics, University of Wisconsin Milwaukee, 3135 N Maryland Ave., Milwaukee, WI 53211; bergda@uwm.edu; erbd@uwm.edu

${ }^{2}$ Minnesota Institute for Astrophysics, University of Minnesota, 116 Church St. SE, Minneapolis, MN 55455; skillman@astro.umn.edu

3 University of Oklahoma; rhenry@ou.edu

${ }^{4}$ Instituto de Astronomía, Universidad Nacional Autónoma de México, Apartado Postal 70-264, Ciudad Universitaria, México DF 04510, México; carigi@astro.unam.mx cal evolution of galaxies. Because $\mathrm{C}$ and $\mathrm{O}$ are important sources of interior opacity in stars, knowledge of the time evolution of CNO abundances is necessary to properly model stellar isochrones. Furthermore, because $\mathrm{C}$ and $\mathrm{O}$ emission lines originate principally in star forming regions, they trace the physical conditions in the gas from which the current generation of mass stars is forming. Equally significant, this interstellar gas carries the signature of the interplay between $\mathrm{SF}$, gas accretion, and supernova-driven feedback across cosmic time.

The $\mathrm{C}$ content in metal-poor star-forming dwarf galaxies was first investigated in detail by Garnett et al. (1995) using Hubble Space Telescope (HST) Faint Object Spectrograph (FOS) observations, who found that $\mathrm{C} / \mathrm{O}$ increases with increasing $\mathrm{O} / \mathrm{H}$. This trend was supported by further UV studies of irregular and spiral galaxies (Garnett et al. 1997; Kobulnicky \& Skillman 1997; Kobulnicky \& Skillman 1998 Garnett et al. 1999; Izotov \& Thuan 1999), implying the apparent secondary ${ }^{4}$ behavior of $\mathrm{C}$ is largely produced by either the time delay in the release of $\mathrm{C}$ by low- and intermediate-mass stars relative to $\mathrm{O}$ or metallicity-dependent yields in massive stars Garnett et al. 1999, Henry et al. 2000, Carigi 2000, Chiappini et al. 2003).

The $\mathrm{C} / \mathrm{O}$ ratio is fairly well studied for stars in the Galactic disk (e.g., Gustafsson et al. 1999, Bensby \&

\footnotetext{
${ }^{4}$ If a particular isotope is produced from the original $\mathrm{H}$ and $\mathrm{He}$ in a star, the production is said to be "primary" and its relative abundance remains constant. If instead the isotope is a daughter element of heavier elements initially present in the star, the production is called "secondary" and is linearly dependent on the initial abundance of the parent heavier elements (metallicity).
} 
Feltzing 2006) and the Galactic halo (e.g., Akerman et al. 2004 Spite et al. 2005, Fabbian et al. 2009), both of which have been found to be consistent with $\mathrm{H}$ II region recombination line (RL) abundances at moderate to high metallicities $(12+\log (\mathrm{O} / \mathrm{H}) \gtrsim 8.0$; e.g., Esteban et al. 2014). However, the monotonic increase in $\mathrm{C} / \mathrm{O}$ with oxygen abundance in metal-poor galaxies reported by Garnett et al. (1995) contrasts with the trend seen in Galactic stars, where the $\mathrm{C} / \mathrm{O}$ ratio is roughly constant over the same range in oxygen abundance. At high redshifts $(\mathrm{z} \gtrsim 1)$, UV $\mathrm{C}$ and $\mathrm{O}$ lines become observable in the optical, opening a window to ground-based $\mathrm{C} / \mathrm{O}$ studies using emission lines from lensed galaxies, composite spectra of large samples, or deep spectra of low metallicity objects in which the $\mathrm{C}$ and $\mathrm{O}$ lines are strong (e.g., Shapley et al. 2003; Erb et al. 2010; James et al. 2014 Stark et al. 2014), as well as absorption lines from metal-poor damped Ly $\alpha$ systems (DLAs). In particular, $\mathrm{C} / \mathrm{O}$ observations of DLAs appear to follow the trend of increasing $\mathrm{C} / \mathrm{O}$ abundance with decreasing metallicity at very low oxygen abundance seen for Milky Way disk stars (e.g., Pettini et al. 2008; Cooke et al. 2011).

\subsection{The Need For Improved UV C/O Measurements}

Carbon, as measured by its bright collisionally excited lines (CELs), has been historically difficult to observe in extragalactic H II regions. Carbon has no strong transitions of its important ionization states in the optical, and there are no IR transitions for $\mathrm{C}^{+2}$, the main ionization state of $\mathrm{C}$, in $\mathrm{H}$ II regions. Other methods have been explored as means to measure $\mathrm{C}$ abundances, such as optical RLs of C II (Esteban et al. 2002; Peimbert et al. 2005; López-Sánchez et al.|2007; García-Rojas \& Esteban 2007; Esteban et al. 2009 Esteban et al. 2014). However, the RLs become very faint at low metallicities; in fact all of the $\mathrm{C} / \mathrm{O}$ measurements from RLs are at oxygen abundances (corrected for temperature fluctuations) above $12+\log (\mathrm{O} / \mathrm{H})=8.0$ (see Esteban et al. 2014, and references therein). Stars provide another way to determine abundances, but this is done with the most luminous stars, which in external galaxies tend to be evolved giants and super giants, whose surfaces are complicated by the effects of internal mixing (Venn 1995).

Little advancement in our understanding has occurred since the insight gained into $\mathrm{C} / \mathrm{O}$ abundances from observations obtained with FOS on HST in the 1990's. In particular, the dispersion in this relationship is unmeasured due to the extremely small sample size. This is largely because few instruments, past and present, have the requisite wavelength coverage and sensitivity, resulting in a paucity of nebular $\mathrm{C} / \mathrm{O}$ measurements in local galaxies, especially at low metallicities. Further, the current body of work is hampered by significant uncertainties due to marginal detections and the difficulties of combining space-based UV and ground-based optical observations (see discussion in Garnett et al. 1995). We can overcome many of these uncertainties and challenges by observing the UV CELs from the dominant ions of $\mathrm{C}$ and $\mathrm{O}$ in ionized nebulae (C III] $\lambda \lambda 1907,1909^{5}$ and O III] $\lambda \lambda 1660,1666)$ with the Cosmic Origins Spectrograph (COS; Green et al. 2012) on HST.

\footnotetext{
5 The UV $\mathrm{C}^{+2}$ doublet, commonly notated as $\mathrm{C} \mathrm{III],} \mathrm{is} \mathrm{actually}$
}

We obtained HST spectroscopic measurements of the UV CELs (O III] $\lambda \lambda 1660,1666$ and C III] $\lambda \lambda 1907,1909)$ in extragalactic H II regions in a sample of targets spanning the metallicity range of dwarf galaxies. We describe our sample selection in Section 2 and details of the UV HST and complimentary optical observations in Sections 3.1 and 3.2 respectively. With the intention of minimizing sources of discrepancies, all data used in this paper were analyzed in a uniform manner as outlined in Section 4. We define criteria for a high-quality $\mathrm{C} / \mathrm{O}$ sample in $\S 5.1$ From these data we investigate the relative $\mathrm{C}$ and $\mathrm{N}$ abundances in Sections 5.2 and 5.3. comparing to RL studies in Section 5.4. In Section 6 we discuss potential sources of carbon. We compare to stellar abundances in $\S 6.1$, discuss potential sources of dispersion in $\S 6.2$, and compare to chemical evolution models in $\S 6.3$. A summary of our results are given in Section 7. The Appendix contains three sections: first, we include the details of the supplemental optical spectra; second, we test the spectrophotometry of the SDSS spectrum for an outlier from our sample by comparing to a follow-up spectrum from the MMT; third, we discuss strategy for future studies.

\section{SAMPLE SELECTION}

The purpose of this study is to obtain new gas-phase UV observations of $\mathrm{C}^{+2}$ and $\mathrm{O}^{+2}$ in a sample of extragalactic $\mathrm{H}$ II regions to aid our understanding of the $\mathrm{C} / \mathrm{O}$ relationship with nebular oxygen abundance. The energies required for ionization to $\mathrm{C}^{+2}$ and $\mathrm{O}^{+2}$ are $24.8 \mathrm{eV}$ and $35.1 \mathrm{eV}$ respectively, and thus these observations are limited to high ionization H II regions. Further, the collisionally excited $\mathrm{C}$ and $\mathrm{O}$ transitions of interest have high excitation energies (6-8 eV), and so are best observed in low metallicity environments where nebular electron temperature $\left(T_{e}\right)$ is high.

Using the C III] $\lambda \lambda 1907,1909 / \mathrm{O}$ III $\lambda 1666$ line ratio is a robust way to investigate the $\mathrm{C} / \mathrm{O}$ relationship for the following reasons (c.f., Garnett et al. 1995): (1) The $\mathrm{C} / \mathrm{O}$ ratio exhibits minimal uncertainty due to reddening, as the interstellar extinction curve is nearly flat over the wavelength range of interest, $1600-2000 \AA$ A. (2) The UV O III] and C III] lines have similar excitation and ionization potentials and so their ratio has little dependence on the physical conditions of the gas, i.e., nebular $T_{e}$ and ionization structure. (3) Measuring the $\mathrm{O}^{+2}$ and $\mathrm{C}^{+2}$ lines simultaneously eliminates the aperture matching and positioning uncertainties that can arise when combining different observational setups.

In order to establish the $\mathrm{C} / \mathrm{O}$ relationship with $\mathrm{O} / \mathrm{H}$ in the sparsely measured metal poor regime, we have chosen objects with high emission line surface brightnesses and low metallicity which span a large range in O abundance. With the completion of the Sloan Digital Sky Survey Data Release $12^{6}$ (SDSS-III DR12; Eisenstein et al. 2011; Alam et al. 2015), many new low-metallicity nearby dwarf galaxies have been identified (e.g., Guseva et al. 2009; Ekta \& Chengalur 2010; Izotov et al. 2012). We culled potential targets from the SDSS observations deemed optimal by several studies:

a combination of the forbidden magnetic quadrupole [C III] $\lambda 1907$ emission line and the semi-forbidden electrodipole C III] $\lambda 1909$ emission line. For ease of notation, we will adopt the simplified, albeit incorrect, form of C III] $\lambda \lambda 1907,1909$. 
1. Izotov et al. (2012) presented 42 low-metallicity emission-line galaxies selected from 7 th data release of the SDSS (Abazajian et al. 2009), including 17 of the most metal-deficient emission-line galaxies known in the local universe at the time.

2. Ekta \& Chengalur (2010) presented 59 blue compact galaxies (BCGs) selected from the 3rd data release of the SDSS (Abazajian et al. 2005) and 32 dwarf irregular galaxies selected from the literature. These 91 galaxies were selected to have accurate gas-phase oxygen abundance determinations.

3. Guseva et al. (2009) presented 44 emission-line galaxies, selected mostly from the 6 th data release of the SDSS (Adelman-McCarthy et al. 2008) as metal-deficient galaxy candidates.

4. The MPA-JHU group ${ }^{7}$ created an emission line data base for the 818,333 unique star-forming targets in the 7th data release of the SDSS.

From these literature sources we set the following criteria for our sample:

1. $12+\log (O / H) \leq 8.2$ : In order to sample the lowmetallicity galaxy population, we considered only targets with direct gas-phase oxygen abundances of $12+\log (\mathrm{O} / \mathrm{H}) \leq 8.2$ based on $[\mathrm{O}$ III $] \lambda 4363$ detections at strengths of $3 \sigma$ or greater.

2. $z<0.26$ : The G140L grating is the only grating on COS that allows simultaneous observations of O III] $\lambda \lambda 1660,1666$ and C III] $\lambda \lambda 1907,1909$ in nearby galaxies. Limited wavelength coverage $(<$ $2405 \AA$ ) and rapidly declining red-ward throughput required targets with redshifts of $z<0.26$.

3. $D_{25} \lesssim 5^{\prime \prime}$ : Through visual inspection of SDSS photometry (Gunn et al. 1998) using the Catalog Archive Server (CAS) database, we selected candidate targets which have compact morphologies in the sense that the diameter of their optical light profiles $\lesssim 5^{\prime \prime}$. This step is important to optimize the sample observations for the $2.5^{\prime \prime}$ COS aperture.

4. $E(B-V)<0.1$ : Only targets with small reddening values were selected, which reduces uncertainties associated with the reddening correction of the UV lines and the relative amount of $\mathrm{C}$ and $\mathrm{O}$ locked up in dust grains.

5. $m_{F U V} \leq 19.5 A B$ : To ensure targets were bright enough in the FUV to enable continuum detections with COS, we required targets to have Galaxy Evolution Explorer (GALEX) photometry (GR6; Bianchi et al. 2014) values of $m_{F U V} \leq 19.5$ AB.

\footnotetext{
6 http://www.sdss.org/dr12/

7 Data catalogues are available from http://www .mpa-garching. mpg.de/SDSS/ The Max Plank institute for Astrophysics/John Hopkins University(MPA/JHU) SDSS data base was produced by a collaboration of researchers(currently or formerly) from the MPA and the JHU. The team is made up of Stephane Charlot (IAP), Guinevere Kauffmann and Simon White (MPA),Tim Heckman (JHU), Christy Tremonti (U. Wisconsin-Madison - formerlyJHU) and Jarle Brinchmann (Leiden University - formerly MPA).
}

6. $E W(5007)>50 \AA$ : To improve upon previous studies which largely lack significant detections of O III] $\lambda 1666$, we selected galaxies with large [O III] $\lambda 5007$ equivalent widths.

7. Predicted $F(O I I I \lambda 1666) S / N>5$ : The GALEX FUV flux within the 2.5" HST/COS aperture was used alongside the optical [O III] line fluxes to select targets for which the COS exposure time calculator (ETC) predicted S/N > 5 in the O III] $\lambda 1666$ line, which is the weakest of the desired UV lines.

As a result of the literature search just described, our final sample contained 12 nearby $(0.003<z<0.135)$, UV-bright $\left(m_{F U V} \leq 19.5 \mathrm{AB}\right)$, compact $\left(\mathrm{D}<5^{\prime \prime}\right)$, lowmetallicity $(12+\log (\mathrm{O} / \mathrm{H}) \leq 8.2)$ dwarf galaxies. Basic properties of our final sample are listed in Table 1 . Assuming solar $M_{B}=5.47$, luminosities are estimated by converting the SDSS $u$ - and $g$-band c-model magnitudes to apparent B-band magnitudes, using the conversion from Jester et al. (2005), before determining the absolute B-band magnitude given the SDSS redshift. Median total stellar masses (following Kauffmann et al. 2003), average total star formation rates (SFR; based on Brinchmann et al. 2004), and average total specific SFRs (sSFR) are taken from the MPA-JHU catalogue. Given our selection of nearby, compact, bright targets, our sample has very low-masses and high sSFRs. Figure 1 displays our sample targets.

\section{SPECTROSCOPIC OBSERVATIONS AND DATA REDUCTION}

\subsection{HST/COS FUV Spectra}

As discussed in Garnett et al. (1995, hereafter G95), the approach described in Section 2 to simultaneously observe $\mathrm{C}$ and $\mathrm{O}$ CELs in the UV has many advantages over studies which combine space-based UV data with ground-based optical data in their $\mathrm{C} / \mathrm{O}$ abundance calculations. This method is currently limited to observations from COS as it is the only space-based UV spectrograph with the necessary sensitivity and wavelength coverage. We therefore acquired 13 orbits of UV spectra of our targets with the COS on the HST as part of HST-GO13312 during cycle 21. To maximize our exposure times, we chose to use a spectroscopic peak search acquisition. As shown in the SDSS images in Figure 1, the central blue bulge of emission in each of our targets falls within a $5^{\prime \prime}$ diameter aperture. Henry et al. (2015) obtained COS spectroscopic observations of 10 Green Pea galaxies (Cardamone et al. 2009), which have similarly compact sizes and morphologies in the SDSS as our sample, and demonstrated that most of the UV continuum emission fell within a central $1^{\prime \prime}$ aperture. Assuming good initial pointing coordinates and successful completion of the acquisition, the COS 2.5" fiber should be well aligned with our targets and capture most of the UV emission.

COS FUV observations were taken in the TIME-TAG mode using the 2.5" PSA aperture and the G140L grating at a central wavelength of $1280 \AA$. In this configuration, segment A has a wavelength range of 1282-2148 $\AA^{8}$, allowing the simultaneous observation of the O III] $\lambda \lambda 1660,1666$ and C III] $\lambda \lambda 1907,1909$ emission lines. We used the FP-POS=ALL setting, which takes 4 images 
TABLE 1

Bright, Compact, Nearby Dwarf Sample

\begin{tabular}{|c|c|c|c|c|c|c|c|c|c|c|c|}
\hline Target & $\begin{array}{c}\text { R.A. } \\
\text { (J2000) }\end{array}$ & $\begin{array}{c}\text { Dec. } \\
(\mathrm{J} 2000)\end{array}$ & $z$ & $\begin{array}{c}M_{B} \\
(\mathrm{mag})\end{array}$ & $\begin{array}{c}\log L_{B} \\
\left(\mathrm{~L}_{\odot}\right)\end{array}$ & $\begin{array}{c}\log M_{\star} \\
\left(\mathrm{M}_{\odot}\right)\end{array}$ & $\begin{array}{l}\log \mathrm{SFR} \\
\left(\mathrm{M}_{\odot} / \mathrm{yr}\right)\end{array}$ & $\begin{array}{c}\log \mathrm{sSFR} \\
\left(\mathrm{yr}^{-1}\right)\end{array}$ & $\begin{array}{c}\text { AB FUV } \\
\text { (mag) }\end{array}$ & $\begin{array}{c}12+\log (\mathrm{O} / \mathrm{H}) \\
(\mathrm{dex})\end{array}$ & $\begin{array}{c}t_{\text {exp }} \\
(\mathrm{s})\end{array}$ \\
\hline J141454 & $14: 14: 54.14$ & $-02: 08: 22.90$ & 0.005 & -13.4 & 7.57 & 6.61 & -1.91 & -8.58 & 19.32 & 7.28 & 4746 \\
\hline J082555 & $08: 25: 55.52$ & $+35: 32: 31.9$ & 0.003 & -12.6 & 7.21 & 6.04 & -1.98 & -8.11 & 18.80 & 7.42 & 2180 \\
\hline J104457 & $10: 44: 57.79$ & $+03: 53: 13.1$ & 0.013 & -15.9 & 8.56 & 6.80 & -0.82 & -7.82 & 18.28 & 7.44 & 2066 \\
\hline J120122 & $12: 01: 22.31$ & $+02: 11: 08.3$ & 0.003 & -12.4 & 7.16 & 6.09 & -1.97 & -8.14 & 18.64 & 7.50 & 2056 \\
\hline J085103 & 08:51:03.64 & $+84: 16: 13.8$ & 0.006 & -9.6 & 6.03 & 6.08 & -2.30 & -8.46 & 17.01 & 7.66 & 2154 \\
\hline J124159 & $12: 41: 59.34$ & $-03: 40: 02.4$ & 0.009 & -14.5 & 7.97 & 6.59 & -1.40 & -8.05 & 19.44 & 7.74 & 1847 \\
\hline J115441 & $11: 54: 41.22$ & $+46: 36: 36.2$ & 0.004 & -13.8 & 7.71 & 6.14 & -2.02 & -8.22 & 17.98 & 7.75 & 2229 \\
\hline $\mathrm{J} 122622$ & $12: 26: 22.71$ & $-01: 15: 12.2$ & 0.007 & -12.4 & 7.15 & 7.21 & -1.10 & -8.37 & 17.67 & 7.77 & 1944 \\
\hline J122436 & $12: 24: 36.71$ & $+37: 24: 36.5$ & 0.040 & -18.0 & 9.41 & 7.86 & -0.06 & -8.01 & 18.41 & 7.78 & 2136 \\
\hline $\mathrm{J} 124827$ & $12: 48: 27.79$ & $+48: 23: 03.3$ & 0.030 & & & 7.47 & -0.49 & -8.05 & 19.44 & 7.80 & 2044 \\
\hline J025426 & $02: 54: 26.12$ & $-00: 41: 22.6$ & 0.015 & -16.4 & 8.77 & 7.65 & -0.67 & -8.40 & 18.54 & 8.06 & 2048 \\
\hline J095137 & 09:51:37.47 & $+48: 39: 41.2$ & 0.135 & -14.8 & 8.13 & 9.34 & 0.49 & -8.93 & 19.10 & 8.20 & 2168 \\
\hline
\end{tabular}

Note. - Selected target sample. All objects are bright, compact, nearby dwarf galaxies with low metallicities as measured by their ground-based optical spectra. The first four columns give the target name used in this work, location, and redshift. Column 5 gives the galaxy B-band AB magnitude, estimated using the conversion of Jester et al. (2005) with the SDSS c-model u and g magnitudes. Luminosity in Column 6 is determined from Column 5 assuming $M_{B, \odot}=5.47$. Note that no SDSS magnitudes are available for J124827. Columns 7-9 list the median total stellar masses, average star formation rates, and average specific star formation rates from the MPA-JHU database. Column 10 lists the FUV magnitudes for these objects from GALEX. Column 11 lists the range of metal-poor direct oxygen abundances of our sample taken from the literature. Column 12 gives the total HST/COS science exposure times.

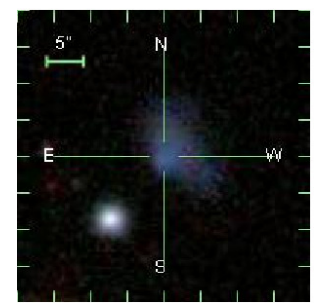

J141454.14-020822.9

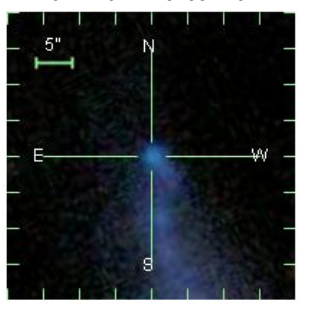

J085103.64+841613.8

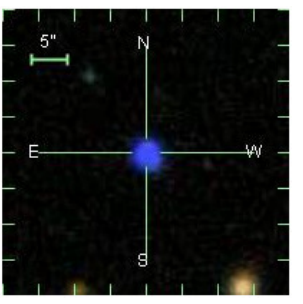

J122436.71+372436.5

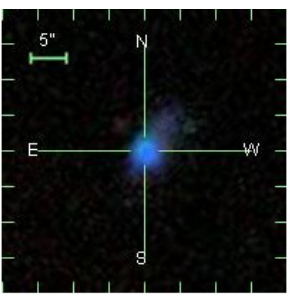

J082555.52+353231.9

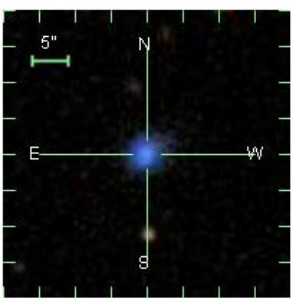

J124159.34-034002.4

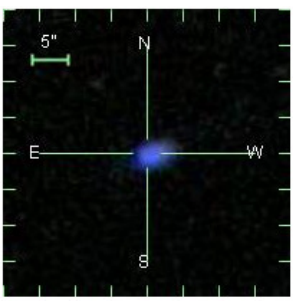

J124827.79+482303.3

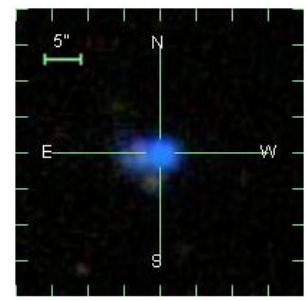

J104457.79+035313.1

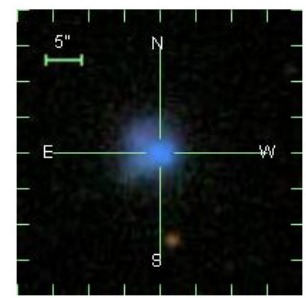

J115441.22+463636

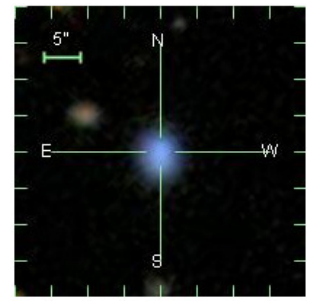

J025426.12-004122.6

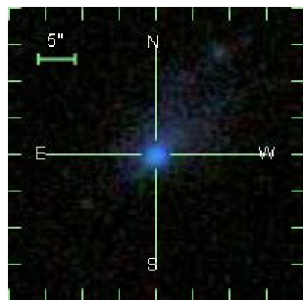

$\mathrm{J} 120122.31+021108.3$

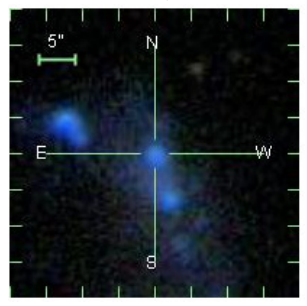

J122622.71-011512.2

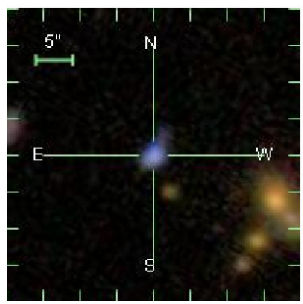

J095137.47+483941.2

FIG. 1. - SDSS images of the 12 targets in our sample. Notice the bright blue appearance and compact morphologies signifying ongoing star formation and high surface brightnesses which allow for maximum flux through the $2.5^{\prime \prime}$ COS aperture.

offset from one another in the dispersion direction, increasing the cumulative $\mathrm{S} / \mathrm{N}$ and mitigating the effects of fixed pattern noise. The 4 positions allow a flat to be created and cosmic rays to be eliminated. Each target was observed for the maximum time allotted in a single orbit as determined by the object orbit visibility, except for J141454 which was prioritized to be observed for 2 orbits to extend the sample to lower metallicities. All data from GO13312 were processed with CALCOS version $2.21^{9}$.

In order to gain signal-to-noise we chose to bin the

8 The G140L grating on COS is characterized as having wavelength coverage out to $2405 \AA$. However, our experience with this setup indicates a range of usefulness out to only $2000 \AA$.

CALCOSReleaseNotes/notes/

http://www.stsci.edu/hst/cos/pipeline/ 
spectra in the dispersion direction. For the G140L grating, six pixels $(80.3 \mathrm{~m} \AA / \mathrm{pix})$ span a resolution element of roughly $0.55 \AA$ at $\lambda 1660$. By measuring individual airglow emission lines in our spectra, we found a typical $\mathrm{FWHM} \approx 3 \AA$, allowing us to re-bin our spectra by the six pixels of a resolution element while maintaining six resolution elements per FWHM.

\subsection{Supporting SDSS Optical Spectra}

Each of the targets in our sample has been previously observed as part of the SDSS DR7. We used the publicly available SDSS data (York et al. 2000), which have been reduced with the SDSS pipeline (Bolton et al. 2012). Preliminary emission line fluxes from the MPAJHU data catalog were used to select these targets such that they had significant [O III] $\lambda 4363$ auroral line detections. However, to ensure uniformity we have remeasured the SDSS emission lines, as described below, and used the most recent atomic data for the subsequent analysis. From the optical spectral line measurements we obtain the physical parameters needed to analyze the UV spectra: interstellar reddening, electron temperature and density, direct oxygen abundance, and degree of ionization of the nebular gas. The details of these calculations are given in Sections 3.3 and 4, with the resulting parameters listed in Tables 2 and 3 .

\subsection{Nebular Emission Line Measurements}

Emission line strengths for both the COS and SDSS spectra were measured using the SPLOT routine within $\mathrm{IRAF}^{10}$. Groups of nearby lines were fit simultaneously, constrained by a single Gaussian FWHM and a single line center offset from the vacuum wavelengths (i.e., redshift). Special attention was paid to the Balmer lines, which can be located in troughs of significant underlying stellar absorption. In the cases where Balmer absorption was clearly visible, the bluer Balmer lines $(\mathrm{H} \delta$ and $\mathrm{H} \gamma$ ) were fit simultaneously with multiple components such that the absorption was fit by a broad, negative Lorentzian profile and the emission was fit by a narrow, positive Gaussian profile. Note that our sample is composed of high ionization star-formation regions that display only weak Balmer absorption, consistent with the hard radiation fields from main sequence $\mathrm{O}$ and $\mathrm{B}$ stars, such that the absorption component is negligible for the stronger $\mathrm{H} \beta$ and $\mathrm{H} \alpha$ emission lines. To ensure that noise spikes are not fit, only emission lines with a strength of $3 \sigma$ or greater are used in the subsequent abundance analysis.

The errors of the flux measurements were approximated using

$$
\sigma_{\lambda} \approx \sqrt{(2 \times \sqrt{N} \times \mathrm{rms})^{2}+\left(0.01 \times F_{\lambda}\right)^{2}},
$$

where $\mathrm{N}$ is the number of pixels spanning the Gaussian profile fit to the narrow emission lines. The rms noise in the continuum was taken to be the average of the rms on each side of an emission line. The two terms in Equation 1 approximate the errors from continuum subtraction and flux calibration. For weak lines, such

10 IRAF is distributed by the National Optical Astronomical Observatories. as the UV CELs, the rms term determines the approximate uncertainty. In the case of strong Balmer, [O III], and other lines, the error is dominated by the inherent uncertainty in the flux calibration and accounted for by adding the $1 \%$ uncertainty of standard star calibrations in CALSPEC (Bohlin 2010).

The COS and SDSS spectra were de-reddened using Balmer line ratios and the application of the Cardelli et al. (1989) reddening law, parametrized by $A_{V}=$ $3.1 E(B-V)$. An initial estimate of the electron temperature was determined from the ratio of the [O III] $\lambda 4363$ auroral line to the [O III] $\lambda \lambda 4959,5007$ nebular lines and used as an input to determine the reddening. We iterate on this process, using the de-reddened [O III] line ratio to determine the new electron temperature, until the change in temperature is less than $10 \mathrm{~K}$. The final reddening estimate is an error weighted average of the individual reddening values determined from the $\mathrm{H} \alpha / \mathrm{H} \beta$, $\mathrm{H} \gamma / \mathrm{H} \beta$, and $\mathrm{H} \delta / \mathrm{H} \beta$ ratios.

All of our targets have low extinction in the range of $\mathrm{E}(\mathrm{B}-\mathrm{V})$ of $\sim 0.05$ to 0.19 . Note that the $\mathrm{C}$ and $\mathrm{O}$ abundances presented here have not been corrected for the fraction of atoms embedded in dust. Peimbert \& Peimbert (2010) have estimated that the depletion of O ranges between roughly $0.08-0.12 \mathrm{dex}$, and has a positive correlation with $\mathrm{O} / \mathrm{H}$ abundance. $\mathrm{C}$ is also expected to be depleted in dust, mainly in polycyclic aromatic hydrocarbons and graphite. The estimates of the amount of $\mathrm{C}$ locked up in dust grains in the local interstellar medium shows a relatively large variation depending on the abundance determination methods applied (see, e.g., Jenkins 2014). For the low abundance targets presented here, and their corresponding small extinctions, the depletion onto dust grains is likely small.

Reddening corrected line intensities measured for the seven objects with significantly detected O III] and C III] are reported in Table 2. Figure 2 shows the rest-frame (corrected by SDSS redshift) $\mathrm{C}$ IV, O III], and C III] emission line regions of the COS spectra for each of the seven targets in our sample with significant $\mathrm{C}$ and $\mathrm{O}$ detections. Note that the spectra of J122426 and J124827 were noticeably noisier than those of the other targets, and so have been displayed with a box-car smoothing of 3 pixels for visual aid. The other five targets in our sample did not have detectable $\mathrm{C}$ or $\mathrm{O}$ emission lines. We discuss the cause of these non-detections in Appendix C and use them to improve our selection criteria for a future sample.

\subsection{Diagnostic Diagrams}

Several of the targets in our sample exhibit strong highionization emission lines. As shown in Figure 2, significant C IV $\lambda \lambda 1548,1550$ and He II $\lambda 1640$ emission is present in 3 of our 7 targets with $\mathrm{C} / \mathrm{O}$ detections. With ionization potentials of $24.6 \mathrm{eV}$ and $47.9 \mathrm{eV}$ to reach He II and C IV respectively, emission line features of these species are more commonly observed in high energy objects, such as AGN. Hainline et al. (2011) used a composite AGN spectrum to show that narrow-lined AGN have very strong $\mathrm{C}$ IV emission on average (EW(C IV) $=16.3 \AA$ ), which is typically much stronger than $\mathrm{C}$ III] (average C IV/C III] flux ratio $\sim 7.5$; Alexandroff et al. $(2013))$. In contrast, we measure much smaller ratios of 
C IV/C III] $(<1.0)$ for our sample, similar to Stark et al. (2014) who observed C IV/C III] in lensed extreme emission line galaxies. Additionally, the He II $\lambda 1640$ emission observed for our sample appears to be narrow, indicative of a nebular origin. Brinchmann et al. (2008) have investigated the origin of nebular He II, arguing that $\mathrm{O}$ stars are the main sources of the He II ionizing photons in metal poor systems with $12+\log (\mathrm{O} / \mathrm{H})<8.0$.

To verify that our target observations originate from photoionized H II regions, we plot four of the standard BPT emission line diagnostic diagrams (Baldwin et al. 1981) in Figure 3. Line measurements for the current sample are plotted as blue points in comparison to the grey locus of SDSS DR7 low-mass $\left(\mathrm{M}_{\star}<10^{9} \mathrm{M}_{\odot}\right.$, which are expected to be relatively low-metallicity following the mass-metallicity relationship; e.g., Tremonti et al. 2004; Berg et al. 2012), star-forming galaxies taken from the MPA-JHU database. In the top two panels, the solid lines are the theoretical starburst limits from Kewley et al. (2006). Based on these plots, our sample exhibits the expected properties of photoionized regions, although they are clearly [N II] and [S II] deficient outliers with respect to average star-forming galaxies. In the bottom panels of Figure 3 we find no indication of contributions from shock excitation, which can manifest as strong $[\mathrm{O} \mathrm{I}]$ emission.

\section{CHEMICAL ABUNDANCES}

In order to minimize sources of uncertainty, we compute the chemical abundances for our sample, as well as the supplemented literature sources (see $\S 5.1$ ), in a uniform, consistent manner. With the exception of the $\mathrm{C} / \mathrm{O}$ ratio, all physical conditions and abundances are calculated from the optical spectra. The $\mathrm{C} / \mathrm{O}$ abundances are determined from the $\mathrm{UV} \mathrm{O}^{+2}$ and $\mathrm{C}^{+2}$ CELs for the reasons given in Sections 1 and 2 .

\subsection{Temperature and Density}

A simple H II region can be modeled by three separate volumes: a low-, intermediate-, and high-ionization zone. Accurate $\mathrm{H}$ II region abundance determinations require reliable electron temperature measurements for each volume. This is typically done by observing a temperature-sensitive auroral-to-strong-line ratio. The [O III] $\mathrm{I}(\lambda \lambda 4959,5007) / \mathrm{I}(\lambda 4363)$ ratio is expected to reflect the temperature in the high ionization zone. We use this ratio to determine electron temperatures using the reddening corrected line-ratios from the SDSS spectra and updated atomic data following Berg et al. (2015), assuming the ions are well-approximated by a 5-level atom $^{11}$. We note that the electron temperature can also be determined from the [O III $] \lambda 5007 / \mathrm{O}$ III $] \lambda 1666$ ratio, as is commonly done in high redshift targets where the intrinsically faint optical auroral line is often undetected. However, for nearby targets, this requires combining space- and ground-based observations, potentially introducing flux matching issues and mismatched aperture effects. In fact, for the seven targets presented here, on average the [O III $] \lambda 5007 / \mathrm{O}$ III $] \lambda 1666$ electron temperatures are $\sim 3000 \mathrm{~K}$ lower than the $[\mathrm{O}$ III] $\lambda 5007 / \lambda 4363$ temperatures. Further observations are needed to understand this difference. For simplicity, we determine the high ionization zone electron temperature from the optical [O III] ratio only.

Once a direct electron temperature is determined, the physical conditions of the other zones are needed to complete the H II region picture. Following Garnett (hereafter G92; 1992), photoionization models can be used to relate the direct temperatures of different ionization zones:

$$
\begin{aligned}
& \mathrm{T}[\mathrm{S} \text { III }]=0.83 \times \mathrm{T}[\mathrm{O} \text { III }]+1700 \mathrm{~K} \\
& \mathrm{~T}[\mathrm{~N} \mathrm{II}]=0.70 \times \mathrm{T}[\mathrm{O} \text { III }]+3000 \mathrm{~K},
\end{aligned}
$$

where Equation 3 was adopted from Campbell et al. (1986), based on models from Stasińska (1982). These relationships are valid for temperatures typical of $\mathrm{H}$ II regions: $T_{e}=2,000-18,000 \mathrm{~K}$. We adopt the [O III] temperature for the high ionization zone, and use the G92 relationships to determine the low- and intermediateionization zone temperatures. We note that several recent studies have investigated the validity of the G92 temperature relationships with respect to updated atomic data, larger samples, and higher quality observations (e.g., Kennicutt et al. 2003, Binette et al. 2012, Berg et al. 2015) and found significant differences. However, by the nature of our target selection, our sample is very high excitation, and so there are minimal contributions from the low-ionization lines ([O II $\lambda \lambda 3727$ and [O II] $\lambda \lambda 7320,7330)$, and thus little dependence on the low-ionization zone temperatures for the oxygen abundances.

The [S II] $\lambda \lambda 6717,6731$ ratio is used to determine the electron densities. Ideally, we would compare with the density determinations from the C III] $\lambda \lambda 1906,1909$ line ratio, but, five of our seven targets have ratios outside of the calibration range of the C III] density diagnostic and the other two have highly discrepant C III] densities. Despite the large uncertainties on the C III] ratios due to the rapidly declining throughput of the G140L grating at those wavelengths, the ratios of J104457 and J122622 fall significantly outside of the theoretically predicted range $(>3 \sigma)$. This optical vs. UV density discrepancy problem has been noted by several studies of high-redshift lensed galaxies (e.g., Hainline et al. 2009, Quider et al. 2009. Christensen et al. 2012; Bayliss et al. 2014), and may be the result of different zones of origination for [S II] and C III] (James et al. 2014). Additionally, the C III] ratio is an insensitive measure of electron density below values of $10^{3} \mathrm{~cm}^{-3}$, in particular, for values of $n_{e} \sim 100 \mathrm{~cm}^{-3}$ that are typical of $\mathrm{H}$ II regions in the local universe. Opportunely, both $\mathrm{C}^{+2}$ and $\mathrm{O}^{+2}$ have high critical densities $\left(>10^{5} / \mathrm{cm}^{3}\right)$ and so are insensitive to electron density. The electron temperature and density determinations are listed in Table 3 .

\subsection{Ionic And Total Abundances}

Ionic abundances relative to hydrogen are calculated using:

$$
\frac{N\left(X^{i}\right)}{N\left(H^{+}\right)}=\frac{I_{\lambda(i)}}{I_{H \beta}} \frac{j_{H \beta}}{j_{\lambda(i)}} .
$$

The emissivity coefficients, $j_{\lambda(i)}$, which are functions of both temperature and density, are determined using a 5-

\footnotetext{
11 https://github.com/moustakas/impro IMPRO is an assortment of routines written in IDL by J. Moustakas for reducing and analyzing multiwavelength imaging and spectroscopy of galaxies.
} 

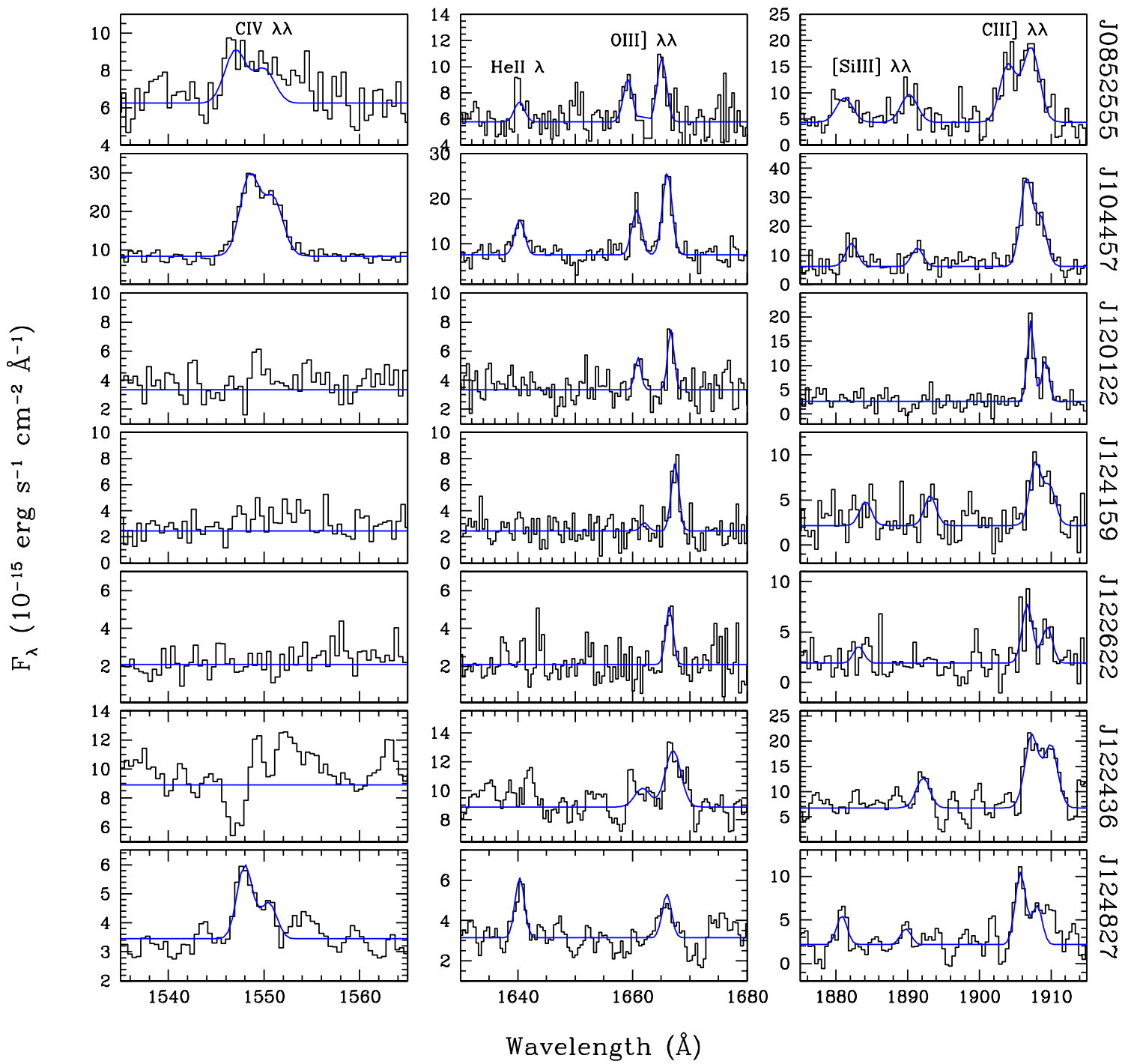

Fig. 2.- HST/COS rest-frame emission line spectra for the seven dwarf galaxies in our sample with significant $\mathrm{C}$ and $\mathrm{O}$ detections. The blue line represents the best fit to the emission lines as described in Section 3.3 Note that the spectra in the last two rows are noisy and so are displayed with a box-car smoothing of 3 .

level atom approximation with the updated atomic data reported in Berg et al. (2015).

Total oxygen abundances $(\mathrm{O} / \mathrm{H})$ are calculated from the simple sum of $\mathrm{O}^{+} / \mathrm{H}^{+}$and $\mathrm{O}^{+2} / \mathrm{H}^{+}$, as contributions from $\mathrm{O}^{+3}$ (requiring an ionization potential of 54.9 $\mathrm{eV})$ are typically negligible in $\mathrm{H}$ II regions. Traditionally, $\mathrm{O}^{+} / \mathrm{H}^{+}$is determined from the optical [O II] $\lambda 3727$ blended line. However, due to the limited SDSS blue wavelength coverage and the redshift of our sample, [O II] $\lambda 3727$ is not detected in 5 of our targets. In these cases, $\mathrm{O}^{+} / \mathrm{H}^{+}$is determined from the optical red [O II] $\lambda \lambda 7320,7330$ doublet (e.g., Kniazev et al. 2004).

Other abundance determinations require ionization correction factors (ICF) to account for unobserved ionic species. For nitrogen, we adopt the convention of N/O $=\mathrm{N}^{+} / \mathrm{O}^{+}($Peimbert 1967), which is valid at a precision of about 10\% (Nava et al. 2006). Multiple ions of sulfur are measured in 5 of our 7 targets. However, unlike the simple ionization structure of nitrogen, both $\mathrm{S}^{+2}$ and $\mathrm{S}^{+3}$ lie in the $\mathrm{O}^{+2}$ zone. To correct for the unobserved
$\mathrm{S}^{+3}$ state, we employ the ICF from Thuan et al. (1995):

$$
\begin{aligned}
\operatorname{ICF}(\mathrm{S}) & =\frac{\mathrm{S}}{\mathrm{S}^{+}+\mathrm{S}^{+2}} \\
& =[0.013+x\{5.10+x[-12.78+x(14.77-6.11 x)]\}]^{-1},
\end{aligned}
$$

where $x=\mathrm{O}^{+} / \mathrm{O}$. Ionic and total $\mathrm{O}, \mathrm{N}$, and $\mathrm{S}$ determined from the optical spectra are listed for our 7 galaxies in Table 3

\subsection{1. $C / O$ Abundance}

In the simplest case, $\mathrm{C} / \mathrm{O}$ can be determined from the $\mathrm{C}^{+2} / \mathrm{O}^{+2}$ ratio alone. Since $\mathrm{O}^{+2}$ has a higher ionization potential than $\mathrm{C}^{+2}(54.9 \mathrm{eV}$ versus $47.9 \mathrm{eV}$, respectively), regions ionized by a hard ionizing spectrum may have a significant amount of carbon in the $\mathrm{C}^{+3}$ form, causing the $\mathrm{C}^{+2} / \mathrm{O}^{+2}$ ionic abundance ratio to underestimate the true $\mathrm{C} / \mathrm{O}$ abundance. The metallicity dependence of the stellar continua, the stellar mass- $T_{\text {eff }}$ 
TABLE 2

Emission-Line Intensities for HSt/COS Observations of Nearby Compact Dwarf Galaxies

\begin{tabular}{|c|c|c|c|c|c|c|c|}
\hline & & & & $I(\lambda) / I(\mathrm{H} \beta)$ & & & \\
\hline Ion & J082555 & J104457 & J120122 & J124159 & J122622 & J122436 & J124827 \\
\hline C IV $\lambda 1548.19$ & $0.55 \pm 0.02$ & $2.07 \pm 0.07$ & & $\ldots$ & & & $0.82 \pm 0.24$ \\
\hline C IV $\lambda 1550.77$ & $0.42 \pm 0.01$ & $1.52 \pm 0.06$ & $\ldots$ & $\ldots$ & $\ldots$ & $\ldots$ & $0.40 \pm 0.24$ \\
\hline He II $\lambda 1640.42$ & $0.38 \pm 0.01$ & $0.70 \pm 0.05$ & $\cdots$ & $\cdots$ & $\cdots$ & $\ldots$ & $0.96 \pm 0.24$ \\
\hline O ІіI] $\lambda 1660.81$ & $0.49 \pm 0.02$ & $0.85 \pm 0.05$ & $0.57 \pm 0.20$ & $0.20 \pm 0.20$ & $\ldots$ & $0.53 \pm 0.11$ & \\
\hline O III $\lambda 1666.15$ & $0.83 \pm 0.02$ & $1.49 \pm 0.06$ & $1.05 \pm 0.20$ & $1.72 \pm 0.28$ & $0.010 \pm 0.001$ & $1.28 \pm 0.11$ & $0.77 \pm 0.22$ \\
\hline Si III $\lambda 1883.00$ & $1.16 \pm 0.03$ & $0.75 \pm 0.06$ & & $1.10 \pm 0.25$ & & & \\
\hline Si III] $\lambda 1892.03$ & $1.25 \pm 0.04$ & $0.66 \pm 0.05$ & & $1.38 \pm 0.25$ & & $1.29 \pm 0.04$ & \\
\hline C III] $\lambda 1906.68$ & $2.63 \pm 0.08$ & $2.83 \pm 0.08$ & $3.89 \pm 0.22$ & $2.94 \pm 0.32$ & $0.022 \pm 0.001$ & $3.38 \pm 0.13$ & $2.12 \pm 0.18$ \\
\hline C III $\lambda 1908.73$ & $3.44 \pm 0.10$ & $1.12 \pm 0.06$ & $2.04 \pm 0.20$ & $1.80 \pm 0.27$ & $0.012 \pm 0.001$ & $2.96 \pm 0.12$ & $1.05 \pm 0.16$ \\
\hline$[\mathrm{O}$ II $] \lambda 3727$ & & $\cdots$ & 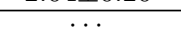 & (1) & & $0.973 \pm 0.029$ & $0.779 \pm 0.025$ \\
\hline$[\mathrm{Ne}$ III $] \lambda 3868$ & $0.276 \pm 0.008$ & & & & $0.485 \pm 0.014$ & & \\
\hline $\mathrm{H} \delta \lambda 4101$ & $0.266 \pm 0.008$ & $0.295 \pm 0.009$ & $0.302 \pm 0.010$ & $0.265 \pm 0.007$ & $0.265 \pm 0.007$ & $0.257 \pm 0.008$ & $0.239 \pm 0.008$ \\
\hline $\mathrm{H} \gamma \lambda 4340$ & $0.476 \pm 0.013$ & $0.506 \pm 0.015$ & $0.513 \pm 0.017$ & $0.495 \pm 0.016$ & $0.493 \pm 0.014$ & $0.458 \pm 0.014$ & $0.470 \pm 0.018$ \\
\hline$[\mathrm{O}$ III] $\lambda 4363$ & $0.116 \pm 0.003$ & $0.146 \pm 0.004$ & $0.103 \pm 0.006$ & $0.105 \pm 0.012$ & $0.116 \pm 0.003$ & $0.111 \pm 0.004$ & $0.129 \pm 0.014$ \\
\hline $\mathrm{H} \beta \lambda 4861$ & $1.000 \pm 0.028$ & $1.000 \pm 0.031$ & $1.000 \pm 0.037$ & $1.000 \pm 0.028$ & $1.000 \pm 0.028$ & $1.000 \pm 0.031$ & $1.000 \pm 0.036$ \\
\hline [O III] $\lambda 4958$ & $1.232 \pm 0.035$ & $1.470 \pm 0.004$ & $1.204 \pm 0.041$ & $1.608 \pm 0.086$ & $1.899 \pm 0.054$ & $1.840 \pm 0.054$ & $1.990 \pm 0.064$ \\
\hline$[\mathrm{O}$ III $] \lambda 5006$ & $3.620 \pm 0.103$ & $4.525 \pm 0.134$ & $3.577 \pm 0.103$ & $4.811 \pm 0.136$ & $5.688 \pm 0.161$ & $5.554 \pm 0.166$ & $5.915 \pm 0.198$ \\
\hline$\left[\begin{array}{l}\mathrm{N} \\
\mathrm{II}\end{array}\right] \lambda 6548$ & $0.005 \pm 0.001$ & $0.003 \pm 0.001$ & & $0.014 \pm 0.004$ & $0.015 \pm 0.001$ & $0.019 \pm 0.003$ & $0.010 \pm 0.012$ \\
\hline $\mathrm{H} \alpha \lambda 6562$ & $2.757 \pm 0.080$ & $2.748 \pm 0.082$ & $2.779 \pm 0.092$ & $2.793 \pm 0.076$ & $2.791 \pm 0.079$ & $2.791 \pm 0.085$ & $2.775 \pm 0.094$ \\
\hline$[\mathrm{N}$ II] $\lambda 6583$ & $0.014 \pm 0.001$ & $0.008 \pm 0.001$ & $0.023 \pm 0.004$ & $0.037 \pm 0.004$ & $0.038 \pm 0.001$ & $0.040 \pm 0.003$ & $0.031 \pm 0.012$ \\
\hline$[\mathrm{S} \mathrm{II}] \lambda 6716$ & $0.026 \pm 0.001$ & $0.022 \pm 0.001$ & $0.052 \pm 0.004$ & $0.098 \pm 0.005$ & $0.106 \pm 0.003$ & $0.087 \pm 0.004$ & $0.084 \pm 0.012$ \\
\hline 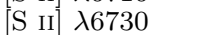 & $0.022 \pm 0.001$ & $0.018 \pm 0.001$ & $0.037 \pm 0.004$ & $0.076 \pm 0.005$ & $0.074 \pm 0.002$ & $0.066 \pm 0.004$ & $0.059 \pm 0.012$ \\
\hline$\left[\begin{array}{lll}\mathrm{O} & \mathrm{II}\end{array} \lambda 7319\right.$ & $0.006 \pm 0.001$ & $0.005 \pm 0.001$ & $0.005 \pm 0.003$ & $0.015 \pm 0.001$ & $0.019 \pm 0.005$ & $0.015 \pm 0.003$ & $0.008 \pm 0.002$ \\
\hline$\left[\begin{array}{lll}\mathrm{O} & \mathrm{II}\end{array}\right] \lambda 7330$ & $0.006 \pm 0.001$ & $0.005 \pm 0.001$ & $0.011 \pm 0.003$ & $0.011 \pm 0.001$ & $0.032 \pm 0.001$ & $0.013 \pm 0.003$ & $0.011 \pm 0.002$ \\
\hline$\left[\begin{array}{l}\mathrm{S} \\
\mathrm{III}\end{array}\right] \lambda 9068$ & $0.045 \pm 0.001$ & $0.040 \pm 0.001$ & $0.063 \pm 0.003$ & $0.082 \pm 0.002$ & $0.103 \pm 0.005$ & & \\
\hline $\mathrm{E}(\mathrm{B}-\mathrm{V})$ & $0.160 \pm 0.009$ & $0.120 \pm 0.010$ & $0.190 \pm 0.010$ & $0.170 \pm 0.008$ & $0.120 \pm 0.009$ & $0.080 \pm 0.010$ & $0.050 \pm 0.012$ \\
\hline $\mathrm{F}_{H \beta}$ & 230.8 & 413.7 & 114.3 & 98.3 & 8131 & 138.4 & 78.1 \\
\hline
\end{tabular}

Note. - The first column lists the vacuum wavelengths of the observed ions for wavelengths of $\lambda<2000 \AA$, and approximate air wavelengths for optical emission lines. The flux values for each object listed are reddening corrected intensity ratios relative to $\mathrm{H} \beta$. The last two rows are extinction and the $\mathrm{H} \beta$ raw fluxes, in units of $10^{-16} \mathrm{erg} \mathrm{s}^{-1} \mathrm{~cm}^{-2}$, measured from the SDSS spectra.
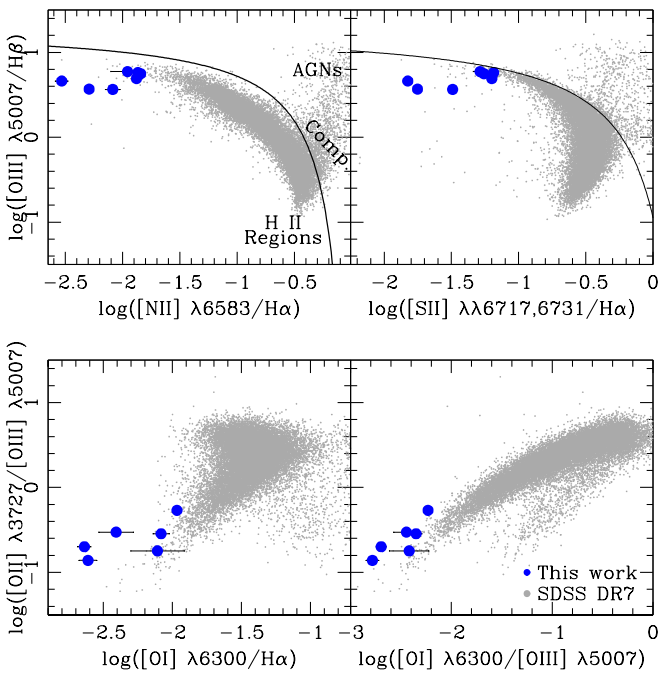

FIG. 3.- SDSS emission line ratios for the seven dwarf galaxies in our sample with significant $\mathrm{C}$ and $\mathrm{O}$ detections. A low-mass subset of the SDSS DR7 is plotted in grey as a comparison sample. The solid lines are the theoretical starburst limits from Kewley et al. (2006).

relation (Maeder 1990), and stellar mass (Terlevich \& Melnick 1985) will also systematically affect the relative ionization tractions of these species. To correct for this effect, we apply the ICF described by G95:

$$
\begin{aligned}
\frac{\mathrm{C}}{\mathrm{O}} & =\frac{\mathrm{C}^{+2}}{\mathrm{O}^{+2}} \times\left[\frac{X\left(\mathrm{C}^{+2}\right)}{X\left(\mathrm{O}^{+2}\right)}\right]^{-1} \\
& =\frac{\mathrm{C}^{+2}}{\mathrm{O}^{+2}} \times \mathrm{ICF},
\end{aligned}
$$

where $\mathrm{X}\left(\mathrm{C}^{+2}\right)$ and $\mathrm{X}\left(\mathrm{O}^{+2}\right)$ are the $\mathrm{C}^{+2}$ and $\mathrm{O}^{+2}$ volume fractions, respectively.

We estimate the ICF as a function of the ionization parameter using CLOUDY (Ferland et al. 2013). Starburst99 models (Leitherer et al. 1999), with and without rotation, for two different metallicities $\left(\mathrm{Z}=0.1 \mathrm{Z}_{\odot}, \mathrm{Z}=\right.$ $0.7 \mathrm{Z}_{\odot}$ ) were considered for a region of continuous star formation. Interestingly, for models assuming a stellar population age of $100 \mathrm{Myr}$, stellar metallicity has a much larger effect on the input ionizing spectrum than including rotation. The ionization fraction of $\mathrm{C}$ and $\mathrm{O}$ species as a function of ionization parameter are shown in Figure 4 for the $\mathrm{Z}=0.1 \mathrm{Z}_{\odot}(\mathrm{Z}=0.002)$ stellar models. In Figure 5 we plot the predicted [O III] $\lambda 5007 /[\mathrm{O}$ II] $\lambda 3727$ emission line ratio versus ionization parameter from our CLOUDY models. We calculated the $\log ([\mathrm{O} \mathrm{III}] /[\mathrm{O} \mathrm{II}])$ ratio and direct oxygen abundance for our sample from the optical spectra and compared to Figure 5 to determine $\log \mathrm{U}$.

The resulting ionization parameters for objects in our sample are plotted against the models in Figures 4 and 5. Vertical dotted lines mark the range of our sample: $-2.48<\log \mathrm{U}<-1.77$, which corresponds with 
a $\mathrm{C}^{+2} / \mathrm{O}^{+2}$ ICF of 0.94-1.16. We estimate the uncertainty in the ICF as the scatter amongst the different models considered (stellar metallicity and rotation effects) at a given $\mathrm{O}^{+2}$ volume fraction. Ionic and total $\mathrm{C}$ abundances, as well as the corrected $\mathrm{C} / \mathrm{O}$ ratio, are given in Table 3 .

Further evidence for the importance of applying an ICF comes from the visible C IV emission in three of our targets in Figure 2. While C IV can be directly measured in these three galaxies, the observed emission doublet is anomalously strong relative to $\mathrm{C} \mathrm{III]} \mathrm{in} \mathrm{comparison} \mathrm{to}$ the CLOUDY model predictions shown in Figure 5 additionally, it can be complicated by stellar contributions. Alternatively, we use the ICF to estimate the $\mathrm{C}^{+3}$ contribution, allowing all seven of our targets to be treated uniformly.

The previous CEL and RL studies compared to in this work have all applied ionization corrections from Garnett et al. (1999). However, our updated photoionization models produce ICFs which agree with the ICFs determined by Garnett et al. (1999) within less than 0.05 dex, on average. Therefore any systematic differences between the CEL and RL abundances presented here or between literature CEL abundances and this work are not related to choice of ICF.

\section{RESULTS}

\subsection{The Optimum $\mathrm{C} / \mathrm{O}$ Sample}

Observations of collisionally excited $\mathrm{C}$ and $\mathrm{O}$ emission exist for only a small number of galaxies. In order to assemble the most comprehensive picture to date of $\mathrm{C} / \mathrm{O}$ determinations, we combine our new observations with previously published values. The first detailed abundance study of the UV spectrum of an $\mathrm{H}$ II region was that of the Orion Nebula by Torres-Peimbert et al. (1980) utilizing International Ultraviolet Explorer (IUE) observations. As reported by Dufour (1984), C/O abundances were measured for perhaps two dozen H II regions with the IUE, yet most were of low signal-to-noise detections and often combined the UV and optical CELs, the exception being the study of N81 by Dufour et al. (1982). Later, the first FOS/HST observations of collisionally excited carbon and oxygen emission lines in the UV were taken by Garnett et al. (1995, 1997) for 7 nearby metalpoor dwarf galaxies. However, O III $\lambda 1666$ was detected at a strength above $3 \sigma$ in only 4 of these targets. Kobulnicky \& Skillman $(1997,1998)$ furthered the study of $\mathrm{C} / \mathrm{O}$, adding measurements in $3 \mathrm{H}$ II regions in NGC 5253 and 3 metal-poor dwarf galaxies respectively. Unfortunately, the UV O III] lines were measured as upper limits, and, consequently, $\mathrm{C} / \mathrm{O}$ abundances were determined using C III] $\lambda 1909$ with [O III] $\lambda 5007$. While Kobulnicky \& Skillman $(1997,1998)$ were careful to use multiple FOS gratings to observe the necessary optical and UV lines with the same instrument and thus avoid aperture effects, the wavelength difference of lines used makes the resulting $\mathrm{C} / \mathrm{O}$ value very sensitive to reddening uncertainties and choice of extinction curve. Izotov \& Thuan (1999) recalculated C/O abundances in a uniform manner for the above studies, but, again, they used the combined UV/optical approach subject to large extinction uncertainties.

The optimal way to constrain the relationship of $\mathrm{C}$
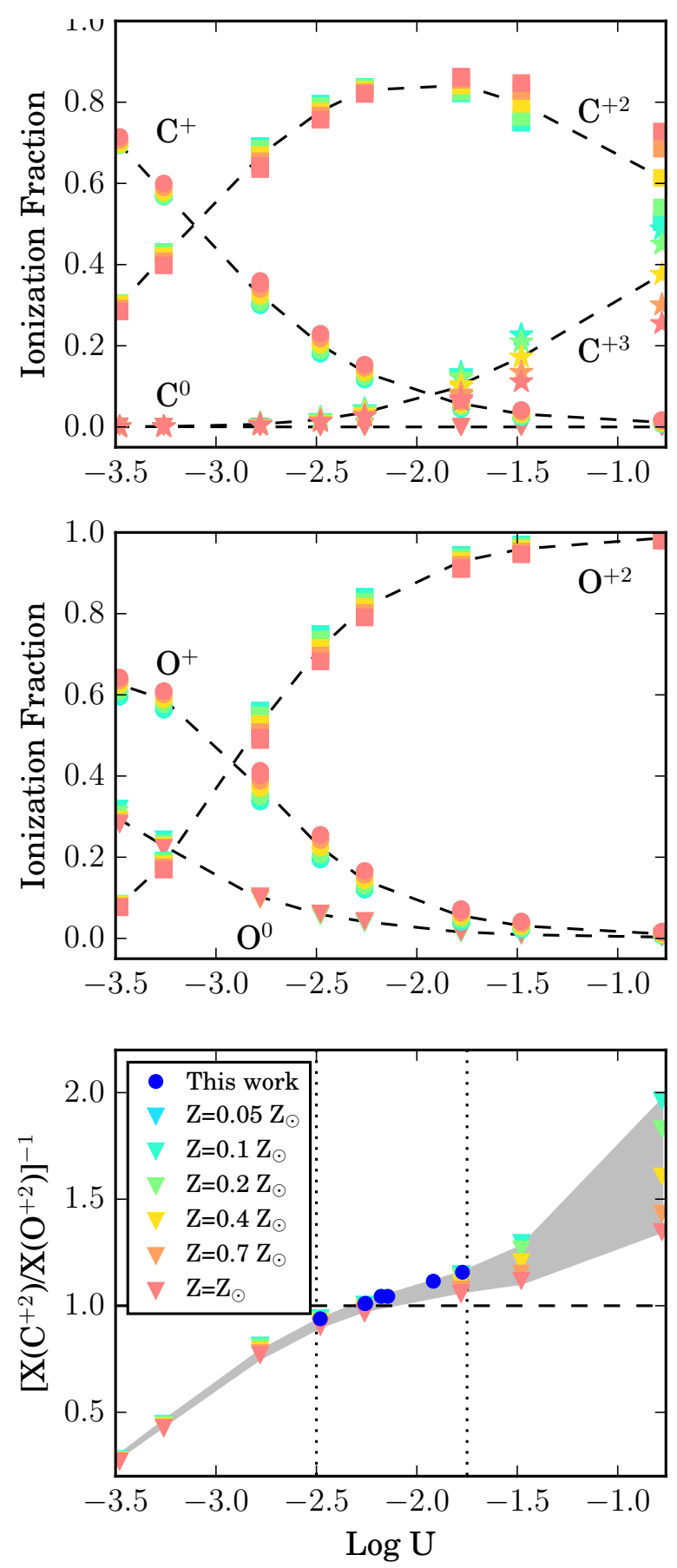

FIG. 4.- The ionization fraction of $\mathrm{C}$ and $\mathrm{O}$ species as a function of ionization parameter for the $\mathrm{Z}=0.1 \mathrm{Z} \odot(\mathrm{Z}=0.002)$ stellar models ignoring rotation. Symbols are color coded by the gasphase oxygen abundance. In the top two panels the different ionic species are designated by triangles, circles, squares, and stars in order of increasing ionization. The bottom panel plots the ionization correction factor versus ionization parameter, where the seven significant detections in our sample are depicted as blue circles and the vertical dotted lines mark the range in log $U$ of our sample. 
TABLE 3

Ionic and Total Abundance for HSt/COS Compact Dwarf Galaxies

\begin{tabular}{|c|c|c|c|c|c|c|c|}
\hline Target & J082555 & J104457 & J120122 & $\mathrm{J} 124159$ & J122622 & J122436 & J124827 \\
\hline \multicolumn{8}{|c|}{ Properties Derived from Optical Spectra } \\
\hline $\mathrm{T}_{e}[\mathrm{O} \mathrm{III}](\mathrm{K})$ & $19,300 \pm 400$ & $19,600 \pm 500$ & $18,300 \pm 600$ & $15,800 \pm 900$ & $15,300 \pm 300$ & 15,20 & 15,800 \\
\hline $\mathrm{T}_{e}[\mathrm{~N} \mathrm{II}](\mathrm{K})^{a}$ & $16,500 \pm 300$ & $16,300 \pm 300$ & $15,800 \pm 400$ & $14,100 \pm 600$ & $13,700 \pm 200$ & $13,600 \pm 200$ & $14,100 \pm 600$ \\
\hline $\mathrm{n}_{e} \mathrm{C}$ III] $\left(\mathrm{cm}^{-3}\right)^{b}$ & 36,600 & 100 & 100 & 100 & 100 & 12,000 & 100 \\
\hline $\mathrm{n}_{e}[\mathrm{~S} \mathrm{II}]\left(\mathrm{cm}^{-3}\right)$ & 260 & 260 & 30 & 150 & 20 & 140 & 10 \\
\hline $\mathrm{O}^{+} / \mathrm{H}^{+}\left(10^{5}\right)$ & 0.18 & $0.17 \pm 0.10$ & $0.36 \pm 0.11$ & $0.87 \pm 0.10$ & $2.15 \pm 0.09$ & $1.13 \pm 0$ & $0.79 \pm 0.08^{c}$ \\
\hline $\mathrm{O}^{+2} / \mathrm{H}^{+}\left(10^{5}\right)$ & 2.19 & $2.62 \pm$ & 2.4 & 4.5 & 5.8 & $5.82 \pm 0.25$ & $5.62 \pm 0.53$ \\
\hline $12+\log (\mathrm{O} / \mathrm{H})$ & $7.37=$ & $7.45 \pm 0.02$ & $7.45 \pm$ & $7.73 \pm 0.04$ & $7.90 \pm 0.01$ & $7.84 \pm 0.02$ & $7.81 \pm 0.03$ \\
\hline $\begin{array}{l}\mathrm{N}^{+} / \mathrm{H}^{+}\left(10^{7}\right) \\
\log (\mathrm{N} / \mathrm{O})\end{array}$ & 0.94 & $0.56 \pm 0.08$ & $1.66 \pm$ & $3.46 \pm$ & & $4.30=$ & $\begin{aligned} 2.80 \pm \\
-1.45+\end{aligned}$ \\
\hline & & & & & & & \\
\hline $\mathrm{S}^{+} / \mathrm{H}^{+}\left(10^{7}\right)$ & 0.45 & $0.37 \pm 0.02$ & 0.85 & $2.10 \pm 0.19$ & $2.23 \pm$ & $1.97 \pm 0.09$ & $1.68 \pm 0.24$ \\
\hline $\mathrm{S}^{+2} / \mathrm{H}^{+}\left(10^{7}\right)$ & $2.66 \pm 0.13$ & $2.30 \pm 0.12$ & $4.03 \pm 0.29$ & $6.37 \pm 0.56$ & $8.42 \pm 0.05$ & $\ldots$ & $\ldots$ \\
\hline $\mathrm{ICF}$ & & 3.825 & 2 & 17 & 1 & .. & . \\
\hline $\log (\mathrm{S} / \mathrm{O})$ & -1.4 & $-1.44 \pm 0$. & -1. & -1.55 & -1.73 & 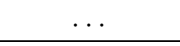 & 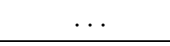 \\
\hline \multicolumn{8}{|c|}{ Properties Derived from UV Spectra } \\
\hline $\mathrm{C}^{+2} / \mathrm{H}^{+}\left(10^{5}\right)$ & $3.12 \pm 0.40$ & $1.91 \pm 0.20$ & $3.90 \pm 1.17$ & $6.25 \pm 2.61$ & $0.053 \pm 0.001$ & $10.5 \pm 1.8$ & $4.20 \pm 1.01$ \\
\hline $\mathrm{C}^{+3} / \mathrm{H}^{+}\left(10^{5}\right)$ & $5.83 \pm 0.13$ & $2.00 \pm 0.19$ & $\ldots$ & $\ldots$ & $\ldots$ & $\ldots$ & $2.34 \pm 0.78$ \\
\hline $\mathrm{O}^{+2} / \mathrm{H}^{+}\left(10^{5}\right)$ & $6.80 \pm 0.16$ & $11.3 \pm 0.07$ & $11.5 \pm 2.3$ & $41.6 \pm 11.6$ & $0.296 \pm 0.001$ & $40.5 \pm 4.5$ & $18.7 \pm 4.1$ \\
\hline $\mathrm{C}^{+2} / \mathrm{O}^{+2}$ & $0.46 \pm 0.06$ & $0.17 \pm 0.02$ & $0.34 \pm 0.09$ & $0.15 \pm 0.07$ & $0.18 \pm 0.02$ & $0.26 \pm 0.05$ & $0.22 \pm 0.10$ \\
\hline $\log \left(\mathrm{C}_{\mathrm{III}}\right] / \mathrm{C}$ IV $)$ & 0.796 & 0.042 & & & & & 0.415 \\
\hline $\log U$ & -1.92 & -1.77 & -2 . & & & -2.26 & -2.15 \\
\hline $\mathrm{ICF}$ & $1.115 \pm 0.085$ & $1.157 \pm 0$ & $1.044 \pm$ & $1.011 \exists$ & $0.939 \pm 0$ & $1.010 \pm 0.040$ & $1.044 \pm 0.045$ \\
\hline $\log (\mathrm{C} / \mathrm{O})$ & $-0.29 \pm 0.06$ & $-0.71 \pm 0.06$ & $-0.45 \pm 0.11$ & $-0.82 \pm 0.16$ & $-0.77 \pm 0.05$ & $-0.59 \pm 0.07$ & $-0.63 \pm 0.17$ \\
\hline
\end{tabular}

Note. - Ionic and total abundance calculations for our compact dwarf galaxy sample. The $\mathrm{T}_{e}[\mathrm{O}$ III] electron temperature, $\mathrm{n}_{e}[\mathrm{~S}$ II] density, and oxygen abundance are determined using the SDSS optical spectra. As shown in Table 2 observed in only two of our seven galaxies, so $\mathrm{O}^{+} / \mathrm{H}^{+}$ionic abundance was determined from the [O II] $\lambda \lambda 7320,7330$ lines unless otherwise notes. $\mathrm{C} / \mathrm{O}$ abundances were calculated using the $\mathrm{C}^{+2} / \mathrm{O}^{+2}$ ratio and corrected for the contribution from other ionization species. The $\mathrm{C} / \mathrm{O}$ ionization parameter is determined using $12+\log (\mathrm{O} / \mathrm{H}), \log ([\mathrm{O}$ III $] /[\mathrm{O}$ II $])$, and the model photoionization diagnostic curves shown in Figures 4 and 5

${ }^{a} \mathrm{~T}_{e}[\mathrm{~N}$ II $]$ determined from $\mathrm{T}_{e}[\mathrm{O}$ III $]$ and the Garnett (1992) relationship.

$\left.{ }^{b} \mathrm{n}_{e} \mathrm{C}_{\mathrm{III}}\right]$ is set equal to 100 when the $\left.\mathrm{C}_{\mathrm{III}}\right] \lambda 1907 / \lambda 1909$ ratio is greater than the low density theoretical limit.

${ }^{c} \mathrm{O}^{+} / \mathrm{H}^{+}$ionic was determined using $[\mathrm{O} \mathrm{II}] \lambda 3727$ line.

with $\mathrm{O}$ is to compare all targets in a uniform way. Because both C III] and O III] are doublet emission lines in the UV, there are a number of ways the observed lines can be combined to determine $\mathrm{C}^{+2} / \mathrm{O}^{+2}$. Past observations with the IUE and HST/FOS have not resolved the C III] $\lambda 1907,1909$ lines, and so these studies used the total C III] $\lambda 1907+\lambda 1909$ intensity in their calculations. While the O III] $\lambda \lambda 1661,1666$ lines can be resolved, the $\lambda 1661$ line is often too weak to detect significantly. Since the ratio of the two O III] lines is physically fixed, nothing is lost in only using the stronger $\lambda 1666$ line in determining the $\mathrm{C} / \mathrm{O}$ ratio. Thus, in order to build upon past studies, we choose to use the UV C III] $\lambda \lambda 1907,1909$ / O III] $\lambda 1666$ line ratio in our $\mathrm{C} / \mathrm{O}$ abundance determinations. Further, we set a minimum criterion of $3 \sigma$ for a line to be considered significantly detected, although we also consider lesser detections in the literature for completeness. Of studies to date, only 5 nearby galaxies meet our best sample criteria of having: (1) UV CEL O III] and C III] detections and (2) an optical direct oxygen abundance, with all relevant lines detected at a strength of $3 \sigma$ or greater. Four of the five targets come from G95: C1543+091, NGC 2363, SBS 0335-052, and SMC N88A (also reported by Kurt et al. 1999), with the addition of N81 from Dufour et al. (1982). Adding these to our sample creates a combined optimal sample of 12 objects.

If we relax the criteria to include less significant O III] $\lambda 1666$ observations, six additional targets can be considered: 30 Dor and T1214-277 from G95, I Zw 18 NW and I Zw 18 SE from Garnett et al. (1997), VS 44 from Garnett et al. (1999), and Mrk 996 from Thuan et al. (1996). For these 11 additional literature targets we compute the $\mathrm{C}$ and $\mathrm{O}$ abundances in a uniform manner following the method outlined in Sections 3 and 4. References for the UV and optical emission line measurements are given in Table 4. Emission line intensities and derived ionic and total abundances for the literature targets are provided in Tables $5-8$

The optimal sample presented here is the largest sample to date of $\mathrm{UV} \mathrm{C} / \mathrm{O}$ abundances calculated in a uniform manner. Note that $\mathrm{C} / \mathrm{O}$ abundances determined from C III] $\lambda \lambda 1907,1909 / \mathrm{O}$ III $] \lambda 1666$ and $\mathrm{C} \mathrm{III]}$ $\lambda \lambda 1907,1909 /[\mathrm{O}$ III] $\lambda 5007$ can differ by up to $0.5 \mathrm{dex}$ in the same source. Since these differences are not systematic and a specific cause has not been identified, we have avoided using $\mathrm{C} / \mathrm{O}$ determinations that use an optical and UV CEL line ratio.

\subsection{Relative $\mathrm{C} / \mathrm{O}$ Abundances}



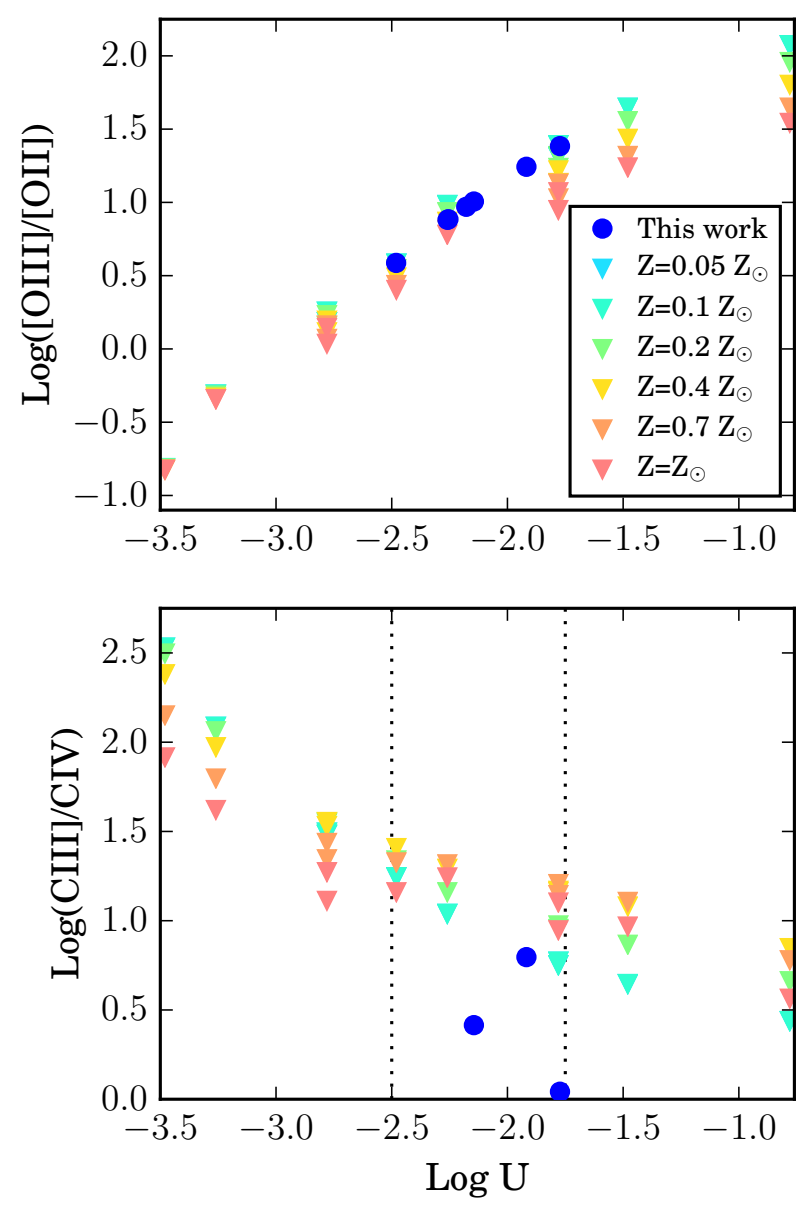

FIG. 5. - Top: $\log ([\mathrm{O}$ III $] \lambda 5007 /[\mathrm{O}$ II] $\lambda 3727)$ versus ionization parameter from CLOUDY models. Symbols are color coded by the gas-phase oxygen abundance. Following the predicted trend for low metallicities allowed us to estimate the ionization parameter for our sample based on the [O III $] \lambda 5007 /[\mathrm{O}$ II $] \lambda 3727$ ratio; the results are plotted as filled blue symbols. Bottom: Predicted $\log (\mathrm{C}$ III $] \lambda \lambda 1907,1909 / \mathrm{C}$ IV $\lambda \lambda 1548,1550)$ UV emission line ratios from CLOUDY models versus ionization parameter. The three targets in our sample with significant C IV emission are plotted as filled blue circles, demonstrating their anomalously strong C IV emission. The range in log $\mathrm{U}$ of our sample is depicted by vertical dotted lines.

The $\mathrm{C} / \mathrm{O}$ abundances for our sample are plotted versus oxygen abundance in the left panel of Figure 6(a). The 12 nearby dwarf galaxies that comprise our optimal sample are plotted as filled blue (this work) and purple (literature sources) circles. For comparison, we have included RL C/O abundances as filled green squares (see Section 5.4.

The dotted line in Figure 6(a) is the least-squares fit to six dwarf galaxies using HST/FOS from G95 (their equation 5), valid for $7.3<12+\log (\mathrm{O} / \mathrm{H})<8.4$. Using this line as a guide, the 12 significant $\mathrm{C} / \mathrm{O}$ detections generally agree with the trend of increasing $\mathrm{C} / \mathrm{O}$ with $\mathrm{O} / \mathrm{H}$, with a few exceptions. In fact, nine of the 12 galaxies are within $2 \sigma$ of the G95 relationship; the exceptions are J082555, J104457, and J120122 from this work. However, without assuming a correlation a priori, the 12 points in our sample in the metallicity range of $7.2 \leq 12+\log (\mathrm{O} / \mathrm{H}) \leq 8.2$ in Figure $6(\mathrm{a})$ demonstrate no trend in $\mathrm{C} / \mathrm{O}$ with $\mathrm{O} / \mathrm{H}$. The dashed line in Figure 6(a) is the weighted mean of the significant UV CEL C/O detections $(\log (\mathrm{C} / \mathrm{O})=-0.62)$, which has a dispersion of $\sigma=0.25$ dex. Visually, the dotted and dashed lines are comparable in their ability to fit the data in that the large dispersion vitiates the presence of any potential real trend. Thus, our uniform sample of $\mathrm{C} / \mathrm{O}$ observations may represent one of two scenarios: either our observations are consistent with the trend found by G95, albeit with outliers, or there is significantly more variability in the $\mathrm{C} / \mathrm{O}$ relationship than previously thought.

The most extreme outlier from the G95 relationship in Figure 6(a) is J082555. We further investigated this target with respect to the $\mathrm{C} / \mathrm{O}$ relationship by remeasuring the dependent variable: $12+\log (\mathrm{O} / \mathrm{H})$. For this purpose, we obtained follow up MMT optical spectra, as described in Appendix C. At the low redshift of J082555, the [ionO2] $\lambda \lambda 3727,3729$ lines are not included in the SDSS spectrum, which prompted further investigation. We find that the SDSS spectrophotometry is consistent with our MMT observations, confirming the low oxygen abundance of J082555. At this oxygen abundance, J082555 appears to be significantly carbon enhanced relative to other metal-poor dwarf galaxies.

In the proceeding sections we discuss the potential sources of this $\mathrm{C} / \mathrm{O}$ distribution and compare to other measures of $\mathrm{C} / \mathrm{O}$, namely RL studies and stellar abundances.

\subsection{Relative $C / N$ Abundances}

In Figure 6(b) we plot the $\mathrm{C} / \mathrm{N}$ ratio versus oxygen abundance using the same symbol designation as Figure 6(a). In this plot, the dashed line represents the weighted mean $\mathrm{C} / \mathrm{N}$ of the 12 targets with significant $\mathrm{C} / \mathrm{O}$ detections. Because there are no significant outliers from this flat relationship to the extent seen in Figure 6(a) our data are consistent with no trend in $\mathrm{C} / \mathrm{N}$ with $\mathrm{O} / \mathrm{H}$. This result contrasts the increasing relationship found by G95.

To reiterate, past studies of nebular $\mathrm{C} / \mathrm{O}$ abundances have found an increasing trend relative to $\mathrm{O} / \mathrm{H}$ with significant dispersion. The updated observations presented here are generally consistent with an increasing relationship, with significant outliers, or could be revealing a plateau with large scatter (similar to the N/O trend seen for dwarf galaxies, e.g., Garnett 1990; Thuan et al. 1995, van Zee et al. 1998; Berg et al. 2012). In early studies of nebular $\mathrm{N} / \mathrm{O}$, an increasing trend with $\mathrm{O} / \mathrm{H}$ was found from observations of a handful of dwarf galaxies. As larger sets of $\mathrm{N} / \mathrm{O}$ data were collected, a different story emerged in which the $\mathrm{N} / \mathrm{O}$ relationship was found to plateau for $12+\log (\mathrm{O} / \mathrm{H}) \lesssim 8$, but with significant scatter. The flat trend seen in Figure 6(b) may indicate that carbon follows a similar pattern to nitrogen.

\subsection{Comparison with Recombination Line Abundances}

The brightest $\mathrm{C}$ and $\mathrm{O}$ recombination lines are $\mathrm{C}$ II $\lambda 4267$ and O II $\lambda 4650$ respectively. While this combination of RLs is relatively insensitive to reddening corrections and electron temperature, optical RLs are inherently faint and therefore observationally challenging, especially at low metallicities. In this regard, UV CELs in low-metallicity galaxies $(12+\log (\mathrm{O} / \mathrm{H})<8.0)$ are complimentary to the high metallicity $\mathrm{RL}$ work. 
The $\mathrm{C} / \mathrm{O}$ relationship has been studied for several nearby galaxies using RLs (Esteban et al. 2002; Peimbert et al. 2005; García-Rojas \& Esteban 2007; López-Sánchez et al. 2007; Esteban et al. 2009; Esteban et al. 2014). In Figures 6(a) and 6(b) we plot the results from these RL studies as filled green squares in comparison to our CEL sample, where the RL observations fill in the $\mathrm{C} / \mathrm{O}$ relationship for $12+\log (\mathrm{O} / \mathrm{H})>8.0$. Esteban et al. (2014) found $\mathrm{C} / \mathrm{O}$ for six extragalactic $\mathrm{H}$ II regions determined from RLs and the $\lambda 1909 / \lambda 5007$ UV-to-optical CEL ratio to be consistent within their uncertainties. Taken together, CELs and RLs seem to follow a general increasing trend in Figure 6(a). However, we caution against forming any conclusions about the trend of $\mathrm{C} / \mathrm{O}$ at low oxygen abundance, as there are too few data points to do so.

Note that for $\mathrm{H}$ II regions, RL absolute abundances are found to be higher than those measured from CELs by up to 0.35 dex (e.g., García-Rojas \& Esteban 2007); this difference is known as the "Abundance Discrepancy Problem". Because RLs and CELs are each useful over a different range in oxygen abundance, we find the benefits of using both RLs and CELs to interpret the $\mathrm{C} / \mathrm{O}$ relationship outweighs this problem. Inspection of Figure 6(a) shows that accounting for the discrepancy by shifting the CEL abundances a few dex toward greater $\mathrm{O} / \mathrm{H}$ values would not alter the appearance of an increasing $\mathrm{C} / \mathrm{O}$ trend.

Based on the data in Figure 6(a), we conclude that $\mathrm{RL} \mathrm{C} / \mathrm{O}$ abundances are more easily observed at higher metallicities (due to their linear dependence on abundance), but show a similar degree of dispersion to CEL abundances in the low metallicity regime. Ideally, we would like to measure the $\mathrm{C} / \mathrm{O}$ relationship over a broad range of oxygen abundance for both CELs and RLs independently. However, given the lack of RL detections in nearby spiral galaxies from $2-6$ hour observations on the Large Binocular Telescope as part of the Chemical Abundances of Spirals project (CHAOS, Berg et al. 2015), this remains a challenging task. Significantly expanding the sample of RL detections will require large integrations times on large telescopes to observe highsurface brightness H II regions in nearby galaxies.

\section{SOURCES OF CARBON}

Carbon is primarily produced by He burning through the triple- $\alpha$ process. However, this reaction can occur in both massive $\left(M>8 M_{\odot}\right)$ and low- to intermediate-mass $\left(1 M_{\odot}<M<8 M_{\odot}\right)$ stars. Previously, the $\mathrm{C} / \mathrm{O}$ ratio has been observed to increase with increasing $\mathrm{O} / \mathrm{H}$ in both individual stars above $12+\log (\mathrm{O} / \mathrm{H})=8.0$ and in galaxies and $\mathrm{H}$ II regions. This trend has been studied by many authors, using a variety of chemical evolution models and stellar yields. Some find that the data are best explained by $\mathrm{C}$ arising almost exclusively from quasi-secondary production in massive stars, in which case the trend in $\mathrm{C} / \mathrm{O}$ versus $\mathrm{O} / \mathrm{H}$ is due to metallicity-dependent stellar winds, as mass loss and ISM enrichment are greater at higher metallicities (Maeder 1992, Henry et al. 2000). Rotation may also play a signiticant role in carbon production. Meynet et al. (2003) showed that rotating stellar models predict enhanced yields of ${ }^{12} \mathrm{C}$ in general, and predict the production of primary ${ }^{13} \mathrm{C}$ at very low metallicity.

Some authors find that carbon production is domi- nated by primary production from low- to intermediatemass stars $\left(1 M_{\odot}<M<3 M_{\odot}\right)$ and that the $\mathrm{C} / \mathrm{O}$ versus $\mathrm{O} / \mathrm{H}$ trend is therefore an evolutionary effect due to the delayed release of carbon relative to oxygen (which is produced almost exclusively by massive stars) in younger and less metal-rich systems (e.g., Chiappini et al. 2003). Carigi et al. (2005) find that massive and low- to intermediate-mass stars contribute roughly equal amounts of carbon in the solar vicinity. Conversely, Akerman et al. (2004) find that the metallicity-dependent mass loss of high mass stars is the main factor in producing the $\mathrm{C} / \mathrm{O}$ versus $\mathrm{O} / \mathrm{H}$ trend, with a small contribution from delayed $\mathrm{C}$ production in lower mass stars.

To examine whether carbon is produced in a similar manner to nitrogen, we refer once again to the plot of $\mathrm{C} / \mathrm{N}$ versus oxygen abundance in Figure 6(b). The relatively constant values of $\mathrm{C} / \mathrm{N}$ across the large range in oxygen abundance suggest that carbon and nitrogen production are dominated by similar mechanisms. For $\mathrm{N} / \mathrm{O}$, a plateau is observed at low oxygen abundance, presumably as a result of primary nitrogen production by intermediate mass stars, whereas secondary nitrogen becomes prominent at higher metallicities $(12+\log (\mathrm{O} / \mathrm{H})$ $>$ 8.3) resulting in an increasing $\mathrm{N} / \mathrm{O}$ ratio with increasing oxygen abundance (Henry et al. 2000). If correct, the flat $\mathrm{C} / \mathrm{N}$ correlation argues for metallicity-independent primary carbon enrichment of the interstellar medium for the low metallicity galaxies investigated here. Quasisecondary carbon production would then become prominent at higher metallicities, but may still play a role at low metallicity, inducing the observed scatter.

\subsection{Comparison with Stellar Abundances}

Another approach to understanding the gas phase carbon abundance is to compare to the abundance pattern of stars, as stellar and nebular abundances are expected to share the same chemical composition once evolutionary effects in stars are accounted for (e.g., Bresolin et al. 2009a: Simón-Díaz \& Stasińska 2011). In Figure 7 we reproduce the same $\mathrm{H}$ II region $\mathrm{RL}$ and $\mathrm{CEL} \mathrm{C} / \mathrm{O}$ abundances from Figure 6(a), but plotted relative to a larger range in $\mathrm{O} / \mathrm{H}$ to allow comparison to $\mathrm{C} / \mathrm{O}$ abundances of Galactic disk stars (4-pointed stars; Gustafsson et al. 1999 ) and halo stars (open triangles; Akerman et al. 2004, Fabbian et al. 2009), as well as metal-poor $(12+\log (\mathrm{O} / \mathrm{H})$ $<7.0$ ) damped Ly $\alpha$ absorbers (DLAs; e.g., Cooke et al. 2011). The sample of Galactic halo stars appear to lie along a plateau just below our sample at low oxygen abundance and with smaller scatter than our extragalactic nebular abundances. However, the elevated $\mathrm{C}$ relative to $\mathrm{O}$ seen in some metal-poor dwarf galaxies could be due to the effects of a significant population of stars with C-rich winds, such as Wolf-Rayet stars.

Large deviations from the $\mathrm{C} / \mathrm{O}$ relationship have been measured for carbon-enhanced metal-poor (CEMP) stars, which are iron poor stars that exhibit elevated carbon $([\mathrm{C} / \mathrm{Fe}] \geq+1.0$ and $[\mathrm{Fe} / \mathrm{H}] \leq-2.0$; Beers \& Christlieb 2005). Interestingly, the frequency of CEMPstars has been observed to increase with declining $[\mathrm{Fe} / \mathrm{H}]$ (e.g., Spite et al. 2013). Of particular interest to our low-metallicity sample is the subclass of CEMP stars, CEMP-no stars, which are classified by their lack of strong neutron-capture-element enhancements and are typically found at lower metallicity than other subclasses 


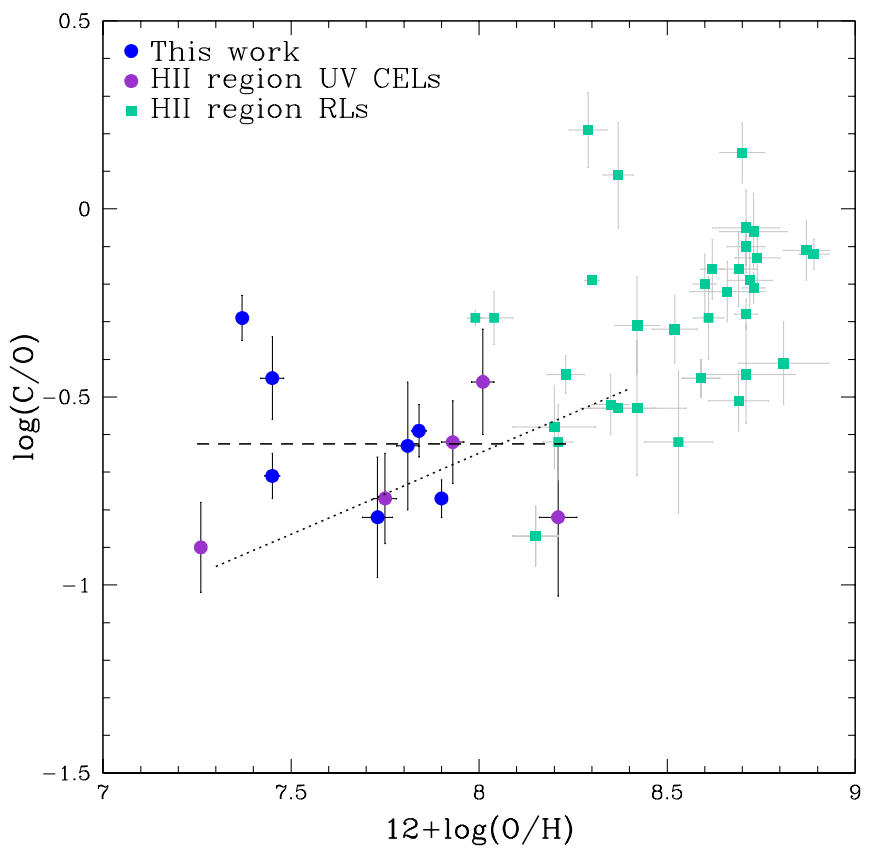

(a)

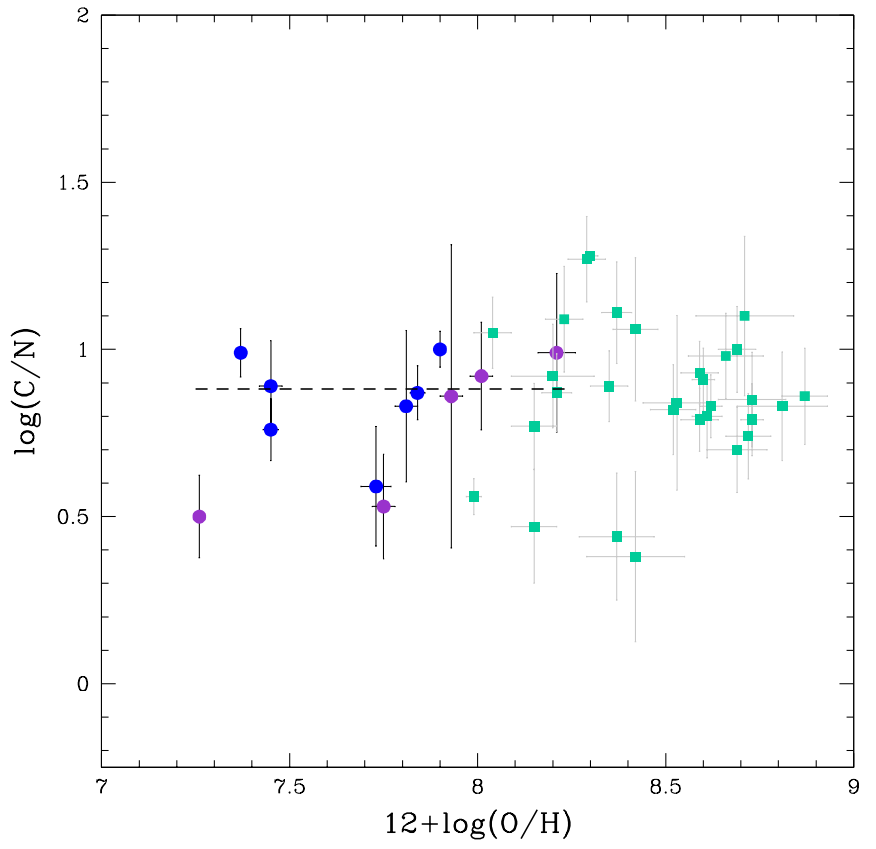

(b)

FIG. 6.- (a) Carbon to oxygen ratio vs. oxygen abundance for star forming galaxies. Our HST/COS observations are plotted as filled blue circles. There are five additional targets in the literature that meet the criteria of having measured direct oxygen abundances and $\mathrm{C} / \mathrm{O}$ abundances determined from UV CELs with strengths of $3 \sigma$ or greater, which are plotted as purple circles. Located at larger oxygen abundances, green filled squares represent star forming galaxies with recombination line abundance determinations (Esteban et al. 2002 Pilyugin \& Thuan 2005 García-Rojas \& Esteban 2007. López-Sánchez et al. 2007 Esteban et al. 2009 Esteban et al. 2014). 'The dotted line is the least-squares fit from G95 and the dashed line is the weighted mean of the significant CEL C/O detections (filled purple and blue circles). (b) Carbon to nitrogen abundance vs. oxygen abundance. While a significant amount of scatter is present $(\sim 0.5$ dex $), \mathrm{C} / \mathrm{N}$ appears to be relatively constant across oxygen abundance, suggesting carbon may follow nitrogen in originating from primary (secondary) production at low (high) values of $\mathrm{O} / \mathrm{H}$. The dashed line marks the weighted mean of the significant CEL C/O detections.

(Aoki et al. 2007). The chemical compositions of CEMPno stars are well matched to the yields of faint, primordial SNe (e.g., Nomoto et al. 2013, Marassi et al. 2014 Tominaga et al. 2014). Under this assumption, first generation / Population III low-energy SNe experienced minimal mixing and large fallback such that the ejection of their outer layers enriched the ISM with Crich, Fe-poor material. CEMP stars then formed as second generation, Fe-deficient stars from the enriched gas. Alternatively, CEMP abundances have also been explained by the chemical contributions of massive metalfree "spinstars", where stellar winds of rapidly-rotating mega metal-poor stars (MMP; $[\mathrm{Fe} / \mathrm{H}]<-6.0)$ with partial mixing eject significant amounts of CNO (Hirschi \& et al. 2006; Meynet et al. 2010, Maeder et al. (2015).

Under the assumption that primordial faint SNe dominated early metal enrichment, Salvadori et al. (2015) investigated the frequency of CEMP stars in 10 Local Group dwarf galaxies with similar masses, luminosities, and gas phase oxygen abundances as the targets in this study (see properties of Local Group dwarf galaxies in Mateo 1998). They found that CEMP-no stars should be present in all dwarf galaxies, but with a relative fraction that is dependent on both luminosity and metallicity. According to this study, the presence of CEMP-no stars relative to the total number of stars can be as significant as $50 \%$ in the most metal-poor $([\mathrm{Fe} / \mathrm{H}]<-4.75)$, lowluminosity $\left(L<10^{4} L_{\odot}\right)$ dwarfs down to $0.02 \%$ in more metal-rich $([\mathrm{Fe} / \mathrm{H}]>-3)$, luminous $\left(L>10^{7} L_{\odot}\right)$ dwarf galaxies. While the chemical evolution history of each galaxy is different, the processes which create CEMP-no stars likely play a varying, but important role in enriching a galaxy's ISM with carbon at early times.

\subsection{Dispersion in $\mathrm{C} / \mathrm{O}$}

Compilations of previous nebular abundance studies have found a linearly increasing $\mathrm{C} / \mathrm{O}$ vs $\mathrm{O} / \mathrm{H}$ relationship with relatively small dispersion, but the simplicity of this relationship may be serendipitous. By recalculating the literature sample in the uniform manner presented in Sections 3 and 4 , we see the trend in $\mathrm{C} / \mathrm{O}$ vs $\mathrm{O} / \mathrm{H}$ (Figure 6(a) shows a weaker correlation than in the original studies. Considering the entire optimal sample presented in Section 5.1, an increasing $\mathrm{C} / \mathrm{O}$ trend emerges that is consistent with G95, but significant scatter (or outliers) is (are) apparent. Garnett (1990) discuss the likelihood of large fluctuations in $\mathrm{C} / \mathrm{O}$ at a fixed $\mathrm{O} / \mathrm{H}$ as a result of delayed $\mathrm{C}$ ejection from intermediate-mass stars of various starburst episodes. Based on the available data of the day, Izotov \& Thuan (1999) found remarkably small dispersion in the $\mathrm{C} / \mathrm{O}$ ratio, claiming it as evidence against time-delayed production of $\mathrm{C}$ in the lowest-metallicity blue compact galaxies.

We have already discussed two potential sources of variations in the $\mathrm{C} / \mathrm{O}$ abundance of dwarf galaxies: the relative fraction of first generation faint SNe dominating early metal-enrichment and the delayed ejection from intermediate-mass stars. Since the star formation histories of dwarf galaxies tend to be bursty (e.g., Lee et al. 
2009), delayed C ejection from intermediate-mass stars of various starburst episodes could result in large fluctuations in $\mathrm{C} / \mathrm{O}$ at a fixed $\mathrm{O} / \mathrm{H}$.

Variations in the initial mass function (IMF) may contribute to the dispersion in carbon abundance at a given oxygen abundance. If both massive and intermediatemass stars are important for carbon production, then variations in the relative number of intermediate-mass to massive stars would also produce significant dispersion. If star formation is triggered in a low-mass cloud, the high mass end of the IMF may be poorly populated (e.g., Fumagalli et al. 2011). Therefore, the ISM enrichment of oxygen by massive stars in the low-mass, metalpoor galaxies studied here could be subject to statistical fluctuations in their IMFs.

A truncation of the upper IMF may also be responsible for the relatively high $\mathrm{C} / \mathrm{O}$ ratios seen in the most metal-poor damped Ly $\alpha$ absorbers with oxygen abundances of $12+\log (\mathrm{O} / \mathrm{H})<7.0$ (e.g., Pettini et al. 2008 . Cooke et al. 2011, Tsujimoto \& Bekki 2011). DLAs from Cooke et al. (2011, orange diamonds) are added to Figure 7. where they follow the trend of Galactic halo stars of increasing $\mathrm{C} / \mathrm{O}$ as $\mathrm{O} / \mathrm{H}$ decreases (e.g., Fabbian et al. 2009). Mattsson (2010) suggest an evolving, top-heavy $\mathrm{IMF}$ during the early stages of Galactic evolution to explain the observed declining trend in $\mathrm{C} / \mathrm{O}$ with increasing $\mathrm{O} / \mathrm{H}$ in the solar neighborhood. Other authors argue for a trend in IMF slope with galaxy mass, in the sense that low-mass galaxies form stars with flatter IMFs (Brown et al. 2012, Kalirai et al. 2013), while galaxies more massive than the Milky Way have steeper IMFs (Conroy \& van Dokkum 2012). Given the interest in possibilities of a non-universal IMF in low-mass metal-poor galaxies, a better characterization of the behavior of $\mathrm{C} / \mathrm{O}$ is vital.

\subsection{Chemical Evolution Models}

We now present an analysis of our $\mathrm{C} / \mathrm{O}$ results for the seven low metallicity dwarf galaxies over a broad metallicity range, where metallicity is assumed to be tracked by oxygen abundance. Figure 7 shows the behavior of $\log (\mathrm{C} / \mathrm{O})$ versus $12+\log (\mathrm{O} / \mathrm{H})$ between roughly 6-9 for the latter parameter. Abundances were taken from studies of several object types, the symbols for which are indicated in the legend. As the value of $12+\log (\mathrm{O} / \mathrm{H})$ increases from 6 to 9 , the data indicate that $\log (\mathrm{C} / \mathrm{O})$ starts at around -0.25 and becomes smaller, declining to a minimum value of roughly -0.9 at $12+\log (\mathrm{O} / \mathrm{H})=$ 7.8. This is followed by a steady increase up to a maximum of 0.0 near $12+\log (\mathrm{O} / \mathrm{H})=8.9$. Note that the positions of our seven low metallicity dwarf galaxies fall in the region in which the observed $\mathrm{C} / \mathrm{O}$ profile passes through its lowest point.

We point out that the clear trends in $\mathrm{C} / \mathrm{O}$ over a broad metallicity range that are exhibited by the data in Figure 7] are produced by an assemblage of abundance measurements of distinctly different object types. The chemical data from these different objects represent the abundance ratios of the interstellar medium at different evolutionary times, where high redshift DLAs are considered to be the progenitors of present day spiral galaxies. Therefore, the presence of an aggregate pattern suggests the existence of a universal thread governing chemical evolution, in spite of the fact that each data point in Figure 7 represents an object whose provenance is unique.
For example, processes related to star formation, as well as mass flows and stellar feedback, are strongly influenced by local conditions within a host galaxy. In turn, these processes can all vary with a galaxy's total mass and rotational properties, as well as local surface densities. Thus, a universal pattern in abundance trends must result from the details of stellar nucleosynthesis which, for the most part, remain robust despite environmental conditions $^{12}$. We have taken this as our working hypothesis.

In an effort to understand the observed profile shape of $\mathrm{C} / \mathrm{O}$ with $\mathrm{O} / \mathrm{H}$ in the context of stellar nucleosynthesis, we compare three model chemical evolution tracks in Figure 8 with the data presented in Figure 7. Each of the tracks represents a one-zone chemical evolution model for the solar vicinity. The three models are taken from papers by Henry et al. (2000, dashed line; HEK), Carigi \& Peimbert (2011, solid line; CP), and Mollá et al. (2015, dotted line; MCGG). The instantaneous recycling approximation was relaxed in all three models, i.e., differences in stellar lifetimes were taken into account when computing the mass of each element released by stars as a function of time. Details of each model can be found in the respective references. Below we provide the salient traits of each for the purpose of this study.

The HEK model included effects of infall (with a timescale of 4 Gyr) but not outflow and employed a metallicity-sensitive star formation efficiency along with the initial mass function of Salpeter (1955). The lower and upper limits on stellar mass were 1 and $120 \mathrm{M}_{\odot}$, respectively. In addition, the massive star $\left(9-120 \mathrm{M}_{\odot}\right)$ yields of Maeder (1992) for carbon, nitrogen, and oxygen were used, while the yields of these same elements for the low and intermediate mass stars (LIMS) of $1-8 \mathrm{M}_{\odot}$ were taken from van den Hoek \& Groenewegen (1997). We note that HEK adjusted their massive star carbon yields slightly upward in order to force agreement with measured $\mathrm{C} / \mathrm{O}$ levels when $12+\log (\mathrm{O} / \mathrm{H})>8.0$.

The MCGG model employed the initial mass function by Kroupa (2001). Their massive star yields for CNO were taken from Limongi \& Chieffi (2003) and Chieffi \& Limongi (2004), while their LIMS yields for those elements were those published by Gavilán et al. (2005. 2006). The lower and upper stellar mass limits were 0.8 and $100 \mathrm{M}_{\odot}$, respectively. Gas infall from the halo to the disk is assumed, with a collapse timescale of $\tau=7.5 \mathrm{Gyr}$ for a galactocentric distance of $8 \mathrm{kpc}$. The star formation process takes place in two steps, with molecular clouds forming first from the diffuse gas, followed by the creation of stars from cloud-cloud collisions. These processes are assumed to occur with efficiencies of 0.40 and 0.20 , respectively. No outflows are assumed in this model.

The CP model considered that the solar vicinity $(r=8$ $\mathrm{kpc}$ ) formed from primordial accretion in a double-infall scenario. The halo was built with a timescale of $\tau=0.5$ Gyr during the first Gyr and then the disk formed with $\tau=8$ Gyr. The star formation rate was similar to the Kennicutt law (Kennicutt 1998), and its efficiency was five times higher during the halo-forming phase than it is

\footnotetext{
12 One important exception to this conclusion may be differences in stellar rotation rates which some stellar evolution models suggest may have a noticeable effect on stellar yields, in particular at low metallicity (Meynet et al. 2006 Hirschi 2007).
} 


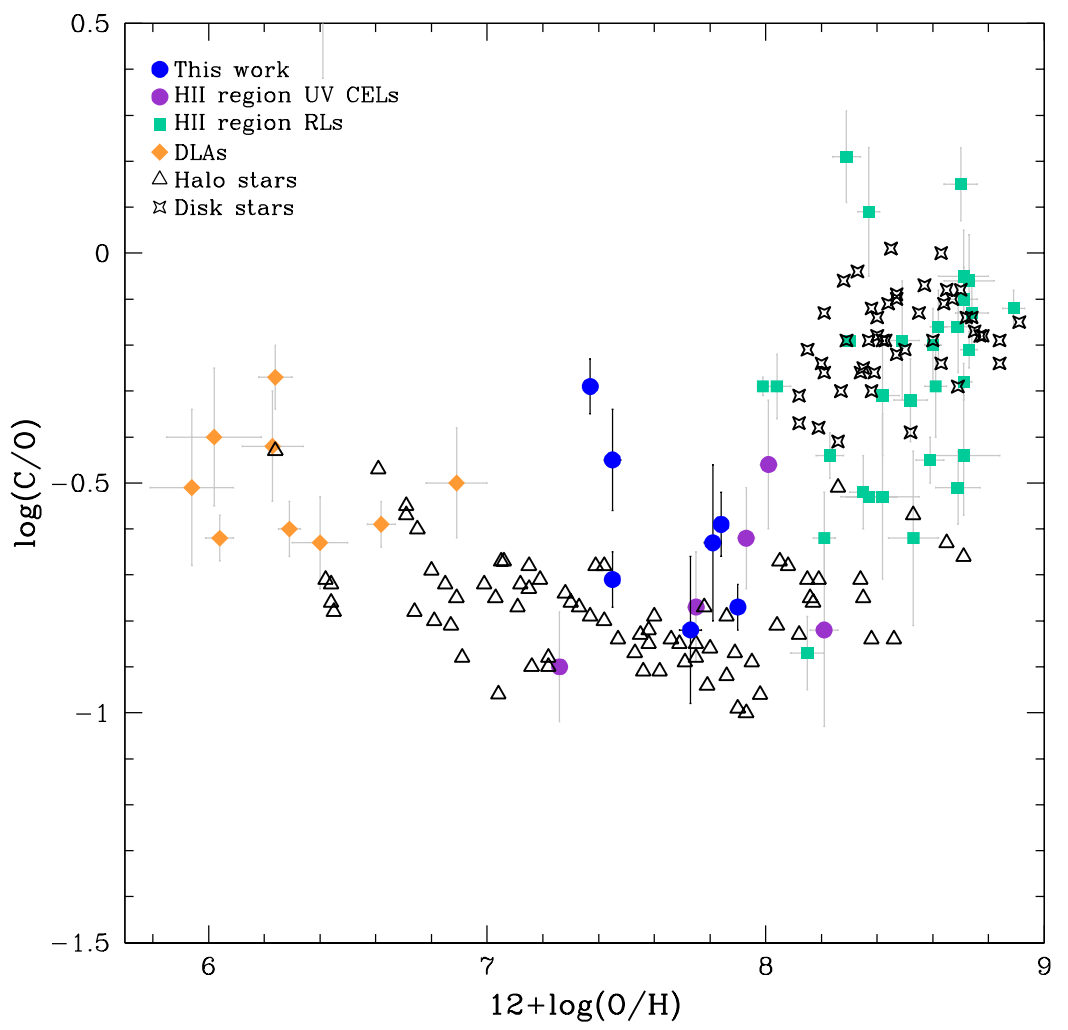

FIG. 7.- Carbon vs. oxygen abundances for a variety of object types. H II region designations are the same as in Figure 6(a) Milky Way stars from both galactic halo star (triangles; Akerman et al. 2004, Spite et al. 2005 Fabbian et al. 2009) and disk star measurements (4-pointed stars; Gustafsson et al. 1999). Damped Lyman alpha systems trom Cooke et al. (2011) to extend coverage to lower oxygen abundances (orange diamonds; Cooke et al. 2011).

during the disk-forming phase. The initial mass function of Kroupa et al. (1993) was applied in the $8-80 M_{\odot}$ range for Pop 111 , and in the $0.08-80 M_{\odot}$ range for all other populations. The metal-dependent yields for $M<8 M_{\odot}$ were taken from Marigo et al. (1996, 1998) and Portinari et al. (1998) and from Hirschil (2007), Meynet \& Maeder (2002), and Maeder (1992, high mass loss rate) for $M>$ $8 \mathrm{M}_{\odot}$. No outtlow was included.

All three models are reasonably consistent with steady increases in $\log (\mathrm{C} / \mathrm{O})$ for $12+\log (\mathrm{O} / \mathrm{H}) \geq 8.1$. For $6.8 \leq$ $12+\log (\mathrm{O} / \mathrm{H}) \leq 8.1$ the tracks from MCGG and CP show little change in $\log (\mathrm{C} / \mathrm{O})$ while HEK's curve rises slightly, all with increasing metallicity. As metallicity declines below $12+\log (\mathrm{O} / \mathrm{H})=6.8$ the curve of HEK falls slightly and that of MCGG remains flat. However, only CP's model track predicts the rise in $\log (\mathrm{C} / \mathrm{O})$ seen for DLAs and halo stars for $12+\log (\mathrm{O} / \mathrm{H})<6.8$.

Clearly, the model by $\mathrm{CP}$ is the most successful at reproducing the details of the observed $\log (\mathrm{C} / \mathrm{O})$ versus $12+\log (\mathrm{O} / \mathrm{H})$ behavior. By considering yields of massive stars that depend on the metallicity as well as rotation, the $\mathrm{CP}$ model is able to predict the inverse relation between these two parameters below $12+\log (\mathrm{O} / \mathrm{H}) \leq 6.8$, in addition to matching the trends for metallicities above this level. In their model, metal-poor massive stars produce low $\mathrm{C} / \mathrm{O}$ yields, but massive stars of high metallicity (and rotating stars with null metallicity) produce high $\mathrm{C} / \mathrm{O}$ yields. In particular, the behavior below 6.8 is due to the early, rapid and high contribution to $\mathrm{C} / \mathrm{O}$ from massive Population III stars whose yield predictions by Hirschi (2007) include the effects of stellar rotation. The plateau at higher metallicities up to $12+\log (\mathrm{O} / \mathrm{H})=$ 8.1 is due to a nearly constant ratio of carbon and oxygen yields from massive Population II stars. At higher metallicities, the semi-loop is due to the primordial infall at the beginning of the disk formation and is followed by the delayed contribution of carbon by Population II LIMS along with a high contribution to $\mathrm{C} / \mathrm{O}$ from Population I massive stars.

While the CP model does an adequate job of matching what we might consider the observed average value of $\log (\mathrm{C} / \mathrm{O})$ in the region between 7.4 and 8.0 in $12+\log (\mathrm{O} / \mathrm{H})$, where our seven low metallicity dwarf galaxies are located, it fails to reproduce the contour of the broad dip in $\log (\mathrm{C} / \mathrm{O})$. As this region is outlined by Galactic halo stars and confirmed to be universal by the presence of both extragalactic H II regions as well as our dwarf galaxies, the dip is most likely due to the behavior of stellar yields of carbon and oxygen in this metallicity range, a behavior that is thus far not predicted by the three yield sets that are tested by the models described here.

One should use caution in using such chemical evolution models to interpret individual objects with contrasting physical properties or directly compare the vastly different samples plotted in Figure 7. It is likely that extant scatter around the trends are related to important differences in events or conditions connected with individual host galaxies. Examples could include feedback, element-selective outflows, variations in the initial 


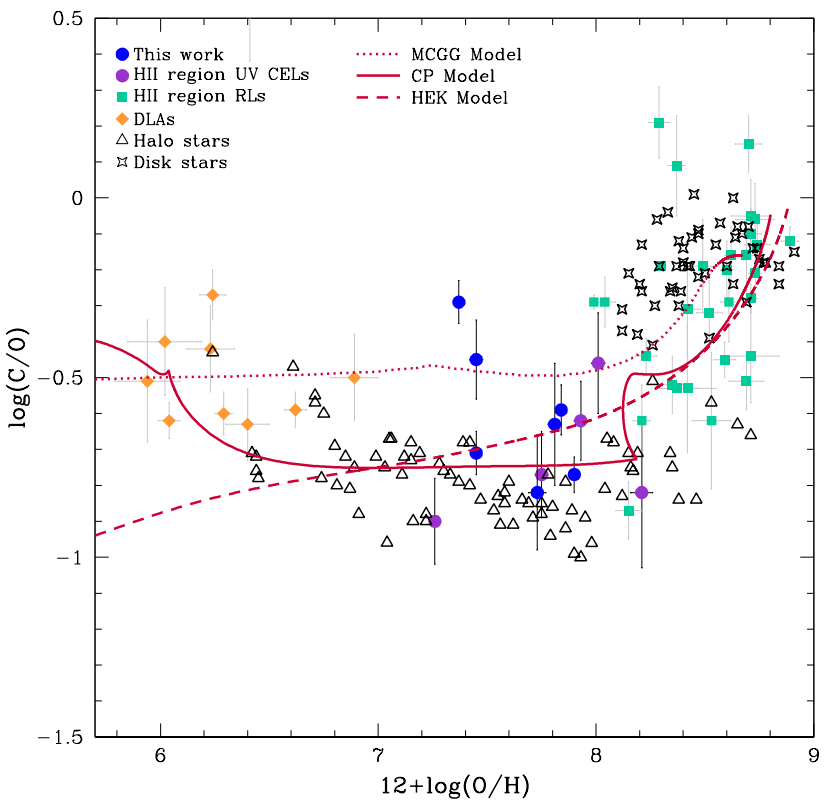

FIG. 8.- Carbon vs. oxygen abundances from Figure 7 in comparison to chemical evolution models from Henry et al. 2000 dashed line), Mollá et al. (2015 dotted line), and Carigi \& Peimbert (2011, solid line). The Carigi \& Peimbert model is the most successful at reproducing the general trend seen for the collective $\log (\mathrm{C} / \mathrm{O})$ data over the observed range in oxygen abundance.

mass function, and the relative fraction of first generation faint SNe dominating early metal-enrichment. For instance, many metal poor stars in dwarf galaxies show abundance ratios similar to the metal poor halo stars (Tolstoy et al. 2009), however, some dwarf galaxies with inferred bursty star formation histories and truncated IMFs may require outflows to reproduce their observed abundance ratios. For this reason, we feel our model analysis based throughout upon the arbitrary choice of conditions in the solar neighborhood is valid for testing the hypothesis that universal patterns of stellar nucleosynthesis serve as the basis for the existence of the trends in $\mathrm{C} / \mathrm{O}$ over a large metallicity range.

In summary, above $12+\log (\mathrm{O} / \mathrm{H})=8.1, \mathrm{C}$ production is apparently governed by a secondary effect in which higher metallicity is responsible for increased $\mathrm{C}$ yields from massive stars. This effect is possibly mediated by an increase in the mass of dust in stellar atmospheres as metallicity increases, resulting in greater wind-driven mass loss.

\section{SUMMARY AND CONCLUSIONS}

To improve our understanding of carbon production, we have obtained carbon and oxygen abundances of 7 nearby, low-metallicity, high-ionization dwarf galaxies using COS on HST. In order to minimize sources of discrepancies and uncertainty, we define our sample with the following criteria:

1. $\mathrm{C} / \mathrm{O}$ abundance is calculated from the UV $\mathrm{C}$ III] $\lambda \lambda 1907,1909$ and O III] $\lambda 1666$ lines such that a single observational setup is used and the reddening law is relatively flat.

2. The nebular physical conditions $-T_{e}, n_{e}, \mathrm{O} / \mathrm{H}-$ are determined using the direct method from complimentary optical observations.

3. All lines are detected at a strength of $3 \sigma$ or greater to reduce spurious detections.

Our $\mathrm{C} / \mathrm{O}$ sample was expanded to include sources from the literature that met the outlined criteria, for which we recalculated the $\mathrm{C} / \mathrm{O}$ and $\mathrm{O} / \mathrm{H}$ abundances to create a uniform sample of 12 objects.

Prior to the present study, the cumulative database of nebular $\mathrm{C} / \mathrm{O}$ abundances of nearby galaxies points to an increasing relationship between $\mathrm{C} / \mathrm{O}$ versus $\mathrm{O} / \mathrm{H}$ abundance (e.g., Garnett et al. 1995). This relationship is based upon samples of few observations and a variety of abundance determination methods, yet is what competing models of low- to intermediate-mass and massive stellar carbon production are based on. With the addition of our new observations at low oxygen abundance, we find that the simple trend of $\mathrm{C} / \mathrm{O}$ increasing with $\mathrm{O} / \mathrm{H}$ is no longer clear due to few measurements with significant scatter. In fact, the current sample, spanning 7.2 $\leq 12+\log (\mathrm{O} / \mathrm{H}) \leq 8.2$, demonstrates no trend in $\mathrm{C} / \mathrm{O}$ at low metallicity. Further observations are needed to determine the trend and dispersion of $\mathrm{C} / \mathrm{O}$ in this metallicity regime.

We examined the $\mathrm{C} / \mathrm{N}$ ratio for our sample and found it to be constant (but with significant scatter) over a large range in oxygen abundance, indicating carbon is predominantly produced by the same nucleosynthetic mechanisms as nitrogen. In this scenario, primary production of carbon results in a flat trend in $\mathrm{C} / \mathrm{O}$ at low metallicity and transitions to an increasing relationship with quasi-secondary production at higher metallicities. If correct, this result, in addition to the flattening predicted by chemical evolution models, would support a constant $\mathrm{C} / \mathrm{O}$ relationship at low nebular metallicities $(12+\log (\mathrm{O} / \mathrm{H}) \leq 8.0)$, in contrast to previous studies.

Potential scenarios to explain the scatter in nebular $\mathrm{C} / \mathrm{O}$ at low metallicity include contributions from both low- to intermediate-mass and high-mass stars, various levels of carbon in a galaxy's primordial chemistry due to low-energy SNe that produced CEMP-no stars, and under populating the high mass end of the IMF. These theories are inherently speculative because they are based on a small sample, and thus larger samples of $\mathrm{UV} \mathrm{C} / \mathrm{O}$ observations are needed. We have established the first UV C/O analysis of local galaxies with COS on HST, and argue that, given the currently available instruments/telescopes, it is the best method to adopt.

DAB and DKE are supported by the US National Science Foundation through the Faculty Early Career Development (CAREER) Program, grant AST-1255591. This work was supported by NASA through grant GO-13312 from the Space Telescope Institute, which is operated by Aura, Inc., under NASA contract NAS5-26555. This paper also used observations obtained at the MMT Observatory, a joint facility of the Smithsonian Institution and the University of Arizona. MMT observations were obtained as part of the University of Minnesota's guaranteed time on Steward Observatory facilities through membership in the Research Corporation and its support for the Large Binocular Telescope. 
TABLE 4

Literature UV and Optical Emission Line Flux Sources

\begin{tabular}{|c|c|c|c|}
\hline \multirow[b]{2}{*}{ Galaxy } & \multirow[b]{2}{*}{$3 \sigma$ Sample? } & \multicolumn{2}{|c|}{ Literature Source } \\
\hline & & UV & Optical \\
\hline N81 & $\checkmark$ & Dufour et al. $(\overline{1982})$ & Dufour et al. $(\overline{1982})$ \\
\hline $\mathrm{C} 1543+091$ & $\checkmark$ & Garnett et al. (1995) & Campbell et al. (1986) \\
\hline NGC 2363 & $\checkmark$ & Garnett et al. (1995) & Pelmbert et al. $(\overline{1986})$ \\
\hline SBS 0335-052E & $\checkmark$ & Garnett et al. (1995) & Izotov et al. (2009) \\
\hline 30 Dor & & Garnett et al. (1995) & Peimbert (2003) \\
\hline T1214-277 & & Garnett et al. (1995) & Guseva et al. (2011) \\
\hline Mrk 996 & & Thuan et al. & Thuan et al. (1996) \\
\hline I Zw $18 \mathrm{NW}$ & & Garnett et aI. (1997) & Skillman \& Kennicutt (1993) \\
\hline I Zw $18 \mathrm{SE}$ & & Garnett et al. (1997) & Skillman \& Kennicutt (1993) \\
\hline VS 44 & & Garnett et al. (1999) & Berg et al. (2013) \\
\hline SMC N88A_barr & $\checkmark$ & Kurt et al. (1999) & Kurt et al. (1999) \\
\hline
\end{tabular}

Funding for the SDSS and SDSS-II has been provided by the Alfred P. Sloan Foundation, the Participating Institutions, the National Science Foundation, the U.S. Department of Energy, the National Aeronautics and Space Administration, the Japanese Monbukagakusho, the Max Planck Society, and the Higher Education Funding Council for England. The SDSS Web Site is http://www.sdss.org/.

The SDSS is managed by the Astrophysical Research Consortium for the Participating Institutions. The Participating Institutions are the American Museum of Natural History, Astrophysical Institute Potsdam, University of Basel, University of Cambridge, Case Western Reserve University, University of Chicago, Drexel University, Fermilab, the Institute for Advanced Study, the Japan Participation Group, Johns Hopkins University, the Joint Institute for Nuclear Astrophysics, the Kavli
Institute for Particle Astrophysics and Cosmology, the Korean Scientist Group, the Chinese Academy of Sciences (LAMOST), Los Alamos National Laboratory, the Max-Planck-Institute for Astronomy (MPIA), the MaxPlanck-Institute for Astrophysics (MPA), New Mexico State University, Ohio State University, University of Pittsburgh, University of Portsmouth, Princeton University, the United States Naval Observatory, and the University of Washington.

This research has made use of NASA's Astrophysics Data System Bibliographic Services and the NASA/IPAC Extragalactic Database (NED), which is operated by the Jet Propulsion Laboratory, California Institute of Technology, under contract with the National Aeronautics and Space Administration.

\section{APPENDIX \\ A. SUPPLEMENTAL OBSERVATIONS}

In Tables $4-8$ we present references for the supplementary UV and optical data used in this work, the emission line intensities adopted from these sources, and the subsequently recalculated ionic and total abundances.

\section{B. FOLLOW-UP OPTICAL MMT SPECTRUM FOR J082555}

Our C/O sample was chosen from the SDSS based on properties of their optical spectra. One of the highest ionization targets from our sample, $\mathrm{J} 082555(\log ([\mathrm{O} \mathrm{III}] /[\mathrm{O} \mathrm{II}])=1.11)$, is also the most significant outlier from the trend of $\mathrm{C} / \mathrm{O}$ with $\mathrm{O} / \mathrm{H}$ in Figures 6(a) 8 . This makes the physical properties of J082555 particularly interesting, and so we want to be confident in the spectrophotometry of the optical spectrum. Additionally, due to the limited blue wavelength coverage of the SDSS spectrograph, many targets in our sample, including J08222, did not have [O II] $\lambda 3727$ line measurements. Instead, the red [O II] $\lambda \lambda 7320,7330$ doublet was used to determine the $\mathrm{O}^{+} / \mathrm{H}^{+}$abundance, despite the fact that it is complicated by lying on top of stellar absorption features as well as lying just inside the blue-end of strong $\mathrm{OH}$ Meinel band emission.

For these reasons we observed a follow-up optical spectrum of J082555 using the Blue Channel spectrograph on the MMT 6.5-m telescope on 11 and 12 November 2015 in order to measure the blue [O II] $\lambda 3727$ emission line and independently confirm the SDSS flux calibration. Three 1200-second exposures were taken for each target with a 1." 5 wide long slit oriented at a position angle equal to the parallactic angle at the midpoint of the observations to minimize differential light loss. The spectra were reduced with the standard techniques outlined in Berg et al. (2012). Emission line flux measurements and chemical abundance calculations were performed in the manner described in Sections 3.3 and 4 respectively.

The resulting de-reddened emission line intensities are given in Table 9, followed by the ionic and total abundances in Table 10. The corresponding SDSS values are tabulated alongside the MMT data for comparison. From Table 9 we conclude that the spectrophotometry of the SDSS and MMT spectra of J082555 are generally well matched. However, there is some difference in the $T_{e}$ in Table 10 resulting in a 0.06 dex in $\log (\mathrm{O} / \mathrm{H})$. Because the $\mathrm{C} / \mathrm{O}$ abundance is determined from the UV spectrum alone (i.e., it is independent of the oxygen abundance determined from the optical spectrum), a much larger offset in $\log (\mathrm{O} / \mathrm{H})$ would be needed for J082555 to no longer be an outlier. 
TABLE 5

Emission-Line Intensities from Literature Sources with C/O Detections

\begin{tabular}{|c|c|c|c|c|c|}
\hline \multirow[b]{2}{*}{ Ion } & \multicolumn{5}{|c|}{$I(\lambda) / I(\mathrm{H} \beta)$} \\
\hline & N81 & C1543+091 & NGC 2363 & SBS0335-052E & SMC N88A_bar \\
\hline O III $] \lambda 1666.15$ & $0.114 \pm 0.036^{a}$ & $0.060 \pm 0.013$ & $0.010 \pm 0.002$ & $0.145 \pm 0.044$ & $0.152 \pm 0.037$ \\
\hline $\mathrm{C}$ III $\lambda 1908.73^{a}$ & $0.302 \pm 0.045$ & $0.175 \pm 0.008$ & $0.045 \pm 0.001$ & $0.261 \pm 0.022$ & $0.904 \pm 0.075$ \\
\hline \begin{tabular}{|l|l|}
$\mathrm{O}$ II & $\lambda 3727$ \\
\end{tabular} & $1.294 \pm 0.183$ & $0.580 \pm 0.021$ & $0.581 \pm 0.023$ & $0.235 \pm 0.006$ & $0.355 \pm 0.027$ \\
\hline [Ne III] $\lambda 3868$ & $0.384 \pm 0.054$ & $0.403 \pm 0.028$ & $0.555 \pm 0.023$ & $0.412 \pm 0.008$ & $0.679 \pm 0.052$ \\
\hline $\mathrm{H} \delta \quad \lambda 4101$ & $0.282 \pm 0.040$ & $0.265 \pm 0.016$ & $0.247 \pm 0.021$ & & $0.294 \pm 0.023$ \\
\hline $\mathrm{H} \gamma \lambda 4340$ & $0.462 \pm 0.065$ & 0.027 & $0.470 \pm 0.022$ & $=0.010$ & $0.479 \pm 0.037$ \\
\hline [O III $] \lambda 4363$ & $0.064 \pm 0.009$ & 0.009 & $0.136 \pm 0.010$ & $=0.003$ & $=0.011$ \\
\hline $\mathrm{H} \beta \lambda 4861$ & $1.000 \pm 0.141$ & $1.000 \pm 0.040$ & $1.000 \pm 0.028$ & $1.000 \pm 0.021$ & $1.000 \pm 0.078$ \\
\hline$[\mathrm{O}$ III] $\lambda 4958$ & $1.768 \pm 0.250$ & $1.932 \pm 0.078$ & $2.342 \pm 0.062$ & $1.095 \pm 0.022$ & $2.623 \pm 0.201$ \\
\hline$[\mathrm{O}$ ІІІ $] \lambda 5006$ & $5.243 \pm 0.742$ & 0.227 & 7.070 & $3.152 \pm 0.065$ & 0.588 \\
\hline$[\mathrm{N}$ II] $\lambda 6548$ & & $0.013 \pm 0.008$ & $0.009 \pm 0.040$ & & $0.015 \pm 0.005$ \\
\hline $\mathrm{H} \alpha \lambda 6562$ & $2.832 \pm 0.400$ & $2.779 \pm 0.093$ & $2.790 \pm 0.075$ & $2.738 \pm$ & $2.805 \pm 0.217$ \\
\hline$[\mathrm{N}$ II $] \lambda 6583$ & $0.062 \pm 0.009$ & $0.032 \pm 0.008$ & $0.023 \pm 0.040$ & $0.009 \pm 0.001$ & $0.036 \pm 0.006$ \\
\hline$[\mathrm{S}$ II] $\lambda 6716$ & $0.031 \pm 0.004$ & $0.049 \pm 0.006$ & $0.044 \pm 0.040$ & $0.017 \pm 0.001$ & $0.012 \pm 0.002$ \\
\hline$\left[\begin{array}{ll}\mathrm{S} \\
\mathrm{II}\end{array}\right] \lambda 6730$ & $0.059 \pm 0.008$ & $0.036 \pm 0.005$ & $0.032 \pm 0.049$ & $0.017 \pm 0.001$ & $0.023 \pm 0.003$ \\
\hline
\end{tabular}

Note. - Emission-line intensities for galaxies supplemented from the literature with $3 \sigma$ O III] and $\mathrm{C}$ III] detections. The first column lists the vacuum wavelengths of the observed ions for wavelengths of $\lambda<2000 \AA$, and approximate air wavelengths for optical emission lines. The flux values for each object listed are reddening corrected intensity ratios relative to $\mathrm{H} \beta$.

${ }^{a}$ Blended line measurement.

TABLE 6

Emission-Line Intensities from Literature Sources with C/O Non-Detections

\begin{tabular}{|c|c|c|c|c|c|c|}
\hline & \multicolumn{6}{|c|}{$\overline{I I(\lambda) / I(\mathrm{H} \beta)}$} \\
\hline Ion & 30 Dor & T1214-277 & Mrk 996 & I Zw $18 \mathrm{NW}$ & I Zw $18 \mathrm{SE}$ & VS 44 \\
\hline \begin{tabular}{l|l}
$\mathrm{O}_{\mathrm{III}}$ & $\lambda 1666.15$
\end{tabular} & $0.111 \pm 0.087$ & $0.007 \pm 0.005$ & $0.401 \pm 0.087$ & $0.270 \pm 0.135$ & $0.290 \pm 0.145$ & $0.017 \pm 0.014$ \\
\hline $\mathrm{C}_{\mathrm{III}} \lambda_{1908.73^{a}}$ & $1.309 \pm 0.045$ & $0.017 \pm 0.002$ & $4.734 \pm 1.980$ & $0.550 \pm 0.091$ & $0.470 \pm 0.071$ & $0.057 \pm 0.007$ \\
\hline \begin{tabular}{ll|l}
$\mathrm{O}$ & $\mathrm{II}$ & $\lambda 3727$
\end{tabular} & $1.526 \pm 0.030$ & $0.308 \pm 0.007$ & $1.029 \pm 0.058$ & $0.264 \pm 0.013$ & $0.466 \pm 0.026$ & $2.470 \pm 0.112$ \\
\hline$[\mathrm{Ne}$ III $] \lambda 3868$ & $0.415 \pm 0.011$ & $0.413 \pm 0.009$ & $0.751 \pm 0.048$ & $0.153 \pm 0.008$ & $0.154 \pm 0.011$ & $0.090 \pm 0.004$ \\
\hline $\mathrm{H} \delta \lambda 4101$ & $0.307 \pm 0.007$ & $0.294 \pm 0.006$ & $0.319 \pm 0.029$ & $0.269 \pm 0.013$ & $0.257 \pm 0.015$ & $0.260 \pm 0.011$ \\
\hline $\mathrm{H} \gamma \lambda 4340$ & $0.529 \pm 0.012$ & $0.509 \pm 0.010$ & $0.530 \pm 0.036$ & $0.469 \pm 0.020$ & $0.435 \pm 0.020$ & $0.480 \pm 0.014$ \\
\hline$[\mathrm{O}$ III $] \lambda 4363$ & $0.035 \pm 0.001$ & $0.173 \pm 0.004$ & $0.220 \pm 0.025$ & $0.062 \pm 0.004$ & $0.044 \pm 0.007$ & $0.008 \pm 0.001$ \\
\hline $\mathrm{H} \beta \lambda 4861$ & $1.000 \pm 0.028$ & $1.000 \pm 0.020$ & $1.000 \pm 0.044$ & $1.000 \pm 0.040$ & $1.000 \pm 0.040$ & $1.000 \pm 0.028$ \\
\hline$[$ O III $] \lambda 4958$ & $1.678 \pm 0.039$ & $1.719 \pm 0.035$ & $0.925 \pm 0.040$ & $0.648 \pm 0.026$ & $0.604 \pm 0.025$ & $0.650 \pm 0.016$ \\
\hline$[\mathrm{O}$ III $] \lambda 5006$ & $5.003 \pm 0.131$ & $5.118 \pm 0.103$ & $2.706 \pm 0.107$ & $1.950 \pm 0.077$ & $1.717 \pm 0.070$ & $1.960 \pm 0.064$ \\
\hline$[\mathrm{N}$ III $\lambda 6548$ & $0.038 \pm 0.001$ & $0.003 \pm 0.001$ & & $0.045 \pm 0.001$ & $0.055 \pm 0.002$ & $0.124 \pm 0.006$ \\
\hline $\mathrm{H} \alpha \lambda 6562$ & $2.859 \pm 0.058$ & $2.750 \pm 0.058$ & $2.701 \pm 0.106$ & $2.760 \pm 0.407$ & $2.760 \pm 0.089$ & $2.910 \pm 0.133$ \\
\hline$[\mathrm{N}$ II $] \lambda 6583$ & $0.115 \pm 0.002$ & $0.008 \pm 0.001$ & $0.153 \pm 0.011$ & $0.008 \pm 0.001$ & $0.013 \pm 0.001$ & $0.300 \pm 0.021$ \\
\hline$[\mathrm{S}$ II] $\lambda 6716$ & $0.071 \pm 0.001$ & $0.022 \pm 0.001$ & $0.052 \pm 0.005$ & $0.024 \pm 0.001$ & $0.041 \pm 0.001$ & $0.210 \pm 0.011$ \\
\hline [S II] $\lambda 6730$ & $0.066 \pm 0.001$ & $0.014 \pm 0.001$ & $0.051 \pm 0.005$ & $0.021 \pm 0.001$ & $0.029 \pm 0.001$ & $0.160 \pm 0.010$ \\
\hline
\end{tabular}

Note. - Same as Table 5 but for galaxies supplemented from the literature with $<3 \sigma$ O III] and $\mathrm{C}$ III] detections.

a Blended line measurement. 
TABLE 7

Ionic and Total Abundance for Literature Sources with C/O Detections

\begin{tabular}{|c|c|c|c|c|c|}
\hline Target & N81 & $\mathrm{C} 1543+091$ & NGC 2363 & SBS0335-052E & SMC N88A_bar \\
\hline \multicolumn{6}{|c|}{ Properties Derived from Optical Spectra } \\
\hline $\begin{array}{l}\mathrm{T}_{e}\left[\begin{array}{ll}\mathrm{O} & \mathrm{III}\end{array}\right](\mathrm{K}) \\
\mathrm{T}_{e}\left[\begin{array}{ll}\mathrm{N} & \mathrm{II}\end{array}\right](\mathrm{K})^{a} \\
\mathrm{n}_{e}\left[\begin{array}{ll}\mathrm{S} & \mathrm{II}\end{array}\right]\left(\mathrm{cm}^{-3}\right)\end{array}$ & $\begin{array}{l}12,200 \pm 800 \\
11,500 \pm 600 \\
9290\end{array}$ & $\begin{array}{c}16,300 \pm 600 \\
14,400 \pm 400 \\
80\end{array}$ & $\begin{array}{c}14,900 \pm 500 \\
13,400 \pm 400 \\
70\end{array}$ & $\begin{array}{c}20,600 \pm 400 \\
17,400 \pm 300 \\
530\end{array}$ & $\begin{array}{c}14,300 \pm 600 \\
13,000 \pm 400 \\
8030\end{array}$ \\
\hline $\begin{array}{l}\mathrm{O}^{+} / \mathrm{H}^{+}\left(10^{5}\right) \\
\mathrm{O}^{+2} / \mathrm{H}^{+}\left(10^{5}\right) \\
12+\log (\mathrm{O} / \mathrm{H})\end{array}$ & $\begin{array}{l}6.25 \pm 1.29 \\
9.92 \pm 1.57 \\
8.21 \pm 0.05\end{array}$ & $\begin{array}{l}0.55 \pm 0.04 \\
5.03 \pm 0.34 \\
7.75 \pm 0.03\end{array}$ & $\begin{array}{l}0.69 \pm 0.05 \\
7.74 \pm 0.56 \\
7.93 \pm 0.03\end{array}$ & $\begin{array}{l}0.14 \pm 0.01 \\
1.68 \pm 0.05 \\
7.26 \pm 0.01\end{array}$ & $\begin{array}{l}1.02 \pm 0.12 \\
9.30 \pm 0.78 \\
8.01 \pm 0.03\end{array}$ \\
\hline $\begin{array}{l}\mathrm{N}^{+} / \mathrm{H}^{+}\left(10^{7}\right) \\
\log (\mathrm{N} / \mathrm{O})\end{array}$ & $\begin{array}{c}9.69 \pm 1.80 \\
-1.81 \pm 0.11 \\
\end{array}$ & $\begin{array}{c}2.80 \pm 0.72 \\
-1.30 \pm 0.10 \\
\end{array}$ & $\begin{array}{c}2.29 \pm 3.99 \\
-1.48 \pm 0.44 \\
\end{array}$ & $\begin{array}{c}0.55 \pm 0.03 \\
-1.40 \pm 0.03 \\
\end{array}$ & $\begin{array}{c}4.25 \pm 0.75 \\
-1.38 \pm 0.08 \\
\end{array}$ \\
\hline \multicolumn{6}{|c|}{ Properties Derived from UV Spectra } \\
\hline $\begin{array}{l}\mathrm{C}^{+2} / \mathrm{H}^{+}\left(10^{5}\right) \\
\mathrm{O}^{+2} / \mathrm{H}^{+}\left(10^{5}\right) \\
\mathrm{C}^{+2} / \mathrm{O}^{+2} \\
\log \mathrm{U} \\
\mathrm{ICF} \\
\log (\mathrm{C} / \mathrm{O})\end{array}$ & $\begin{array}{c}1.83 \pm 0.08 \\
15.8 \pm 0.6 \\
0.12 \pm 0.07 \\
-2.50 \\
0.933 \\
-0.82 \pm 0.21\end{array}$ & $\begin{array}{c}0.20 \pm 0.01 \\
1.25 \pm 0.02 \\
0.16 \pm 0.05 \\
-2.16 \\
1.049 \\
-0.77 \pm 0.12\end{array}$ & $\begin{array}{c}0.08 \pm 0.01 \\
0.36 \pm 0.01 \\
0.22 \pm 0.06 \\
-2.07 \\
1.074 \\
-0.62 \pm 0.11\end{array}$ & $\begin{array}{c}0.10 \pm 0.01 \\
0.88 \pm 0.04 \\
0.12 \pm 0.04 \\
-2.03 \\
1.085 \\
-0.90 \pm 0.12\end{array}$ & $\begin{array}{c}2.07 \pm 0.15 \\
4.86 \pm 0.18 \\
0.43 \pm 0.14 \\
-1.83 \\
1.141 \\
-0.46 \pm 0.14\end{array}$ \\
\hline
\end{tabular}

TABLE 8

Ionic and Total Abundance for Literature Sources with C/O Non-Detections

\begin{tabular}{|c|c|c|c|c|c|c|}
\hline Target & 30 Dor & T1214-277 & Mrk 996 & I Zw $18 \mathrm{NW}$ & I $\mathrm{Zw} 18 \mathrm{SE}$ & VS 44 \\
\hline \multicolumn{7}{|c|}{ Properties Derived from Optical Spectra } \\
\hline $\begin{array}{l}\mathrm{T}_{e}\left[\begin{array}{ll}\mathrm{O} & \mathrm{III}\end{array}\right](\mathrm{K}) \\
\mathrm{T}_{e}\left[\begin{array}{ll}\mathrm{N} & \mathrm{II}\end{array}\right](\mathrm{K})^{a} \\
\mathrm{n}_{e}\left[\begin{array}{ll}\mathrm{S} & \mathrm{II}\end{array}\right]\left(\mathrm{cm}^{-3}\right)\end{array}$ & $\begin{array}{c}10,200 \pm 100 \\
10,200 \pm 200 \\
500\end{array}$ & $\begin{array}{c}20,100 \pm 300 \\
17,000 \pm 200 \\
100\end{array}$ & $\begin{array}{c}25,000 \pm 1000^{a} \\
20,500 \pm 200 \\
620\end{array}$ & $\begin{array}{c}19,400 \pm 900 \\
16,600 \pm 600 \\
330\end{array}$ & $\begin{array}{c}17,000 \pm 1500 \\
14,900 \pm 1000 \\
50\end{array}$ & $\begin{array}{c}8,700 \pm 200 \\
9,100 \pm 200 \\
130\end{array}$ \\
\hline $\begin{array}{l}\mathrm{O}^{+} / \mathrm{H}^{+}\left(10^{5}\right) \\
\mathrm{O}^{+2} / \mathrm{H}^{+}\left(10^{5}\right) \\
12+\log (\mathrm{O} / \mathrm{H})\end{array}$ & $\begin{array}{c}5.72 \pm 0.36 \\
16.6 \pm 0.4 \\
8.35 \pm 0.01\end{array}$ & $\begin{array}{l}0.18 \pm 0.01 \\
2.87 \pm 0.07 \\
7.48 \pm 0.01\end{array}$ & $\begin{array}{l}0.41 \pm 0.02 \\
1.01 \pm 0.05 \\
7.15 \pm 0.02\end{array}$ & $\begin{array}{l}0.17 \pm 0.02 \\
1.17 \pm 0.08 \\
7.13 \pm 0.03\end{array}$ & $\begin{array}{l}0.39 \pm 0.06 \\
1.39 \pm 0.21 \\
7.25 \pm 0.05\end{array}$ & $\begin{array}{c}14.3 \pm 1.2 \\
11.9 \pm 0.7 \\
8.42 \pm 0.02\end{array}$ \\
\hline $\begin{array}{l}\mathrm{N}^{+} / \mathrm{H}^{+}\left(10^{7}\right) \\
\log (\mathrm{N} / \mathrm{O})\end{array}$ & $\begin{array}{c}22.3 \pm 1.2 \\
-1.41 \pm 0.03 \\
\end{array}$ & $\begin{array}{c}0.54 \pm 0.02 \\
-1.52 \pm 0.02 \\
\end{array}$ & $\begin{array}{c}7.16 \pm 0.63 \\
-0.76 \pm 0.04 \\
\end{array}$ & $\begin{array}{c}0.55 \pm 0.05 \\
-1.50 \pm 0.05 \\
\end{array}$ & $\begin{array}{c}1.08 \pm 0.15 \\
-1.56 \pm 0.08 \\
\end{array}$ & $\begin{array}{c}79.0 \pm 7.4 \\
-1.26 \pm 0.05 \\
\end{array}$ \\
\hline \multicolumn{7}{|c|}{ Properties Derived from UV Spectra } \\
\hline $\begin{array}{l}\mathrm{C}^{+2} / \mathrm{H}^{+}\left(10^{5}\right) \\
\mathrm{O}^{+2} / \mathrm{H}^{+}\left(10^{5}\right) \\
\mathrm{C}^{+2} / \mathrm{O}^{+2} \\
\log \mathrm{U} \\
\mathrm{ICF} \\
\log (\mathrm{C} / \mathrm{O})\end{array}$ & $\begin{array}{c}28.0 \pm 1.3 \\
68.0 \pm 5.9 \\
0.41 \pm 0.32 \\
-2.57 \\
0.899 \\
-0.43 \pm 0.25\end{array}$ & $\begin{array}{c}0.007 \pm 0.001 \\
0.045 \pm 0.001 \\
0.16 \pm 0.12 \\
-1.94 \\
1.109 \\
-0.74 \pm 0.25\end{array}$ & $\begin{array}{c}0.90 \pm 1.79 \\
1.07 \pm 0.09 \\
0.85 \pm 0.42 \\
-2.65 \\
0.861 \\
-0.14 \pm 0.18\end{array}$ & $\begin{array}{c}0.28 \pm 0.03 \\
2.21 \pm 0.30 \\
0.13 \pm 0.07 \\
-2.27 \\
1.017 \\
-0.89 \pm 0.20\end{array}$ & $\begin{array}{c}0.43 \pm 0.03 \\
4.65 \pm 0.67 \\
0.93 \pm 0.66 \\
-2.53 \\
0.918 \\
-1.07 \pm 0.23\end{array}$ & $\begin{array}{c}4.82 \pm 0.03 \\
51.6 \pm 0.7 \\
0.09 \pm 0.08 \\
-3.04 \\
0.620 \\
-1.24 \pm 0.27\end{array}$ \\
\hline
\end{tabular}


TABLE 9

COMPARISON OF EMISSION-Line InTENSITIES FROM SDSS AND MMT OBSERVATIONS OF J082555

\begin{tabular}{|c|c|c|}
\hline & \multicolumn{2}{|c|}{$I(\lambda) / I(\mathrm{H} \beta)$} \\
\hline Ion & SDSS & MMT \\
\hline$\left[\begin{array}{ll}\mathrm{O} & \mathrm{II}\end{array} \lambda 3727\right.$ & $\cdots$ & $0.286 \pm 0.006$ \\
\hline $\mathrm{H} 12 \lambda 3750$ & $\ldots$ & $0.020 \pm 0.001$ \\
\hline H11 $\lambda 3770$ & $\cdots$ & $0.028 \pm 0.001$ \\
\hline H10 $\lambda 3797$ & $\ldots$ & $0.042 \pm 0.004$ \\
\hline He I $\lambda 3819$ & $\cdots$ & $.009 \pm 0.001$ \\
\hline H9 $\lambda 3835$ & $0.076 \pm 0.002$ & $0.062 \pm 0.002$ \\
\hline$[\mathrm{Ne}$ III $] \lambda 3868$ & $317 \pm 0.009$ & $0.288 \pm 0.06$ \\
\hline $\mathrm{He} \mathrm{I}+\mathrm{H} 8 \lambda 3889$ & $230 \pm 0.006$ & $0.206 \pm 0.004$ \\
\hline$[\mathrm{Ne}$ III $] \lambda 3967+\mathrm{H} 7$ & $0.203 \pm 0.006$ & $0.245 \pm 0.006$ \\
\hline$[\mathrm{Ne}$ III $] \lambda 4011$ & & $0.003 \pm 0.002$ \\
\hline He I $\lambda 4026$ & $0.020 \pm 0.001$ & $0.018 \pm 0.001$ \\
\hline [S II $] \lambda 4068$ & & \\
\hline $\mathrm{H} \delta \lambda 4$ & $74 \pm 0$ & $0.251 \pm 0.005$ \\
\hline $\mathrm{He}$ & & 0.00 \\
\hline $\mathrm{He}$ & . & 02 \\
\hline $\mathrm{H} \gamma \lambda 4340$ & $0.486 \pm 0.014$ & $0.455 \pm 0.009$ \\
\hline$[\mathrm{O}$ III] $\lambda$ & $0.115 \pm 0.003$ & $0.105 \pm 0.003$ \\
\hline He I $\lambda 43$ & & $0.004 \pm 0.001$ \\
\hline He I $\lambda 4471$ & $0.038 \pm 0.001$ & $0.036 \pm 0.001$ \\
\hline [Ar IV] $\lambda 4711$ & $011 \pm 0$. & \\
\hline$[$ Ar IV $] \lambda 4740$ & $009 \pm 0$ & \\
\hline $\mathrm{H} \beta \lambda 4861$ & $0 \pm 0$ & $.000 \pm 0.029$ \\
\hline He I $\lambda 4921$ & $010 \pm 0.001$ & $0.008 \pm 0.001$ \\
\hline [O III] $\lambda 4958$ & $1.236 \pm 0.025$ & $1.201 \pm 0.034$ \\
\hline$[\mathrm{O} \mathrm{III}] \lambda 50$ & $4 \pm 0$ & $3.708 \pm 0.105$ \\
\hline He I $\lambda 5015$ & $0.018 \pm 0.001$ & $0.024 \pm 0.001$ \\
\hline NI $\lambda 519$ & $\ldots$ & $=0.001$ \\
\hline $\mathrm{Cl}$ & $\ldots$ & $0.003 \pm 0.001$ \\
\hline $\mathrm{Cl}$ & $\ldots$ & $0.002 \pm 0.001$ \\
\hline He I $\lambda 5875$ & & \\
\hline$\left[\begin{array}{lll}\mathrm{O} & 1\end{array}\right] \lambda 6300$ & $0.006 \pm 0.001$ & $0.005 \pm 0.001$ \\
\hline [S III] $\lambda 6312$ & $0.010 \pm 0.001$ & $0.009 \pm 0.001$ \\
\hline 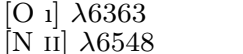 & $\begin{array}{c}\cdots \\
04 \pm 0\end{array}$ & 01 \\
\hline $\mathrm{H} \alpha \lambda 6562$ & $4 \pm 0$ & \pm 0.083 \\
\hline$[\mathrm{N} \mathrm{II]} \lambda 6583$ & $0.013 \pm 0.001$ & $0.013 \pm 0.001$ \\
\hline He I $\lambda 6678$ & $0.024 \pm 0.001$ & $0.027 \pm 0.002$ \\
\hline$[\mathrm{S}$ II $] \lambda 6716$ & $0.026 \pm 0.001$ & $0.028 \pm 0.002$ \\
\hline$\left[\begin{array}{l}\mathrm{S} \\
\mathrm{II}\end{array}\right] \lambda 6730$ & $0.022 \pm 0.001$ & $0.019 \pm 0.002$ \\
\hline [O II] $\lambda 7320$ & $0.006 \pm 0.001$ & $\cdots$ \\
\hline [O II] $\lambda 7330$ & $0.006 \pm 0.001$ & $\cdots$ \\
\hline$E(B-V)$ & $0.160 \pm 0.009$ & $0.000 \pm 0.012$ \\
\hline $\mathrm{F}_{H \beta}$ & 230.8 & 108.9 \\
\hline
\end{tabular}

Note. - The first column lists the vacuum wavelengths of the observed ions for wavelengths of $\lambda<2000 \AA$, and approximate air wavelengths for optical emission lines. The flux values are the reddening corrected intensity ratios relative to $\mathrm{H} \beta$. The last two rows are the extinction and the $\mathrm{H} \beta$ raw flux, in units of $10^{-16} \mathrm{erg} \mathrm{s}^{-1} \mathrm{~cm}^{-2}$

\section{IMPROVED SAMPLE SELECTED FOR FUTURE STUDIES}

It is difficult to predict the $\mathrm{S} / \mathrm{N}$ of emission lines in UV spectra without preliminary UV spectra. Within our COS sample we have examples of successful $\mathrm{C} / \mathrm{O}$ detections and non-detections alike. Contrasting this set of objects allows us to determine what parameters predict successful observations of significant $\mathrm{C}$ and $\mathrm{O}$. We examined many properties, including GALEX magnitude, redshift, reddening, and optical emission line fluxes, but found no correlation with detection of the UV CELs. However, excitation (indicated by $\log [\mathrm{O} \mathrm{III}] \lambda 5007 /[\mathrm{O}$ II $] \lambda 3727$ ) does play a significant role. This is not surprising as both $\mathrm{O} \mathrm{III}]$ and $\mathrm{C}$ III] require high excitation (and ionization) energies. However, the SDSS spectrograph has a wavelength coverage of 3800-9200 $\AA$ and so [O II] $\lambda 3727$ was only observed in 4 of our 12 targets (when $z>0.02$ ).

The importance of high excitation is depicted in Figure 9(a) where we have plotted our C/O sample on the BPT diagram. Once again, significant $\mathrm{C} / \mathrm{O}$ measurements are depicted as closed circles, whereas low $\mathrm{S} / \mathrm{N}$ and non-detections are shown as open circles. It is striking that all of our significant COS detections lie in the upper left hand corner, which we highlight with a yellow box. Since the x-axis of the BPT diagram is correlated with increasing oxygen abundance, 
TABLE 10

Comparison of Ionic and Total Abundance

FROM SDSS AND MMT OBSERVATIONS OF J082555

\begin{tabular}{lcc}
\hline \hline & SDSS & MMT \\
\hline $\mathrm{T}_{e}[\mathrm{O} \mathrm{III}](\mathrm{K})$ & $19,300 \pm 400$ & $18,200 \pm 300$ \\
$\mathrm{~T}_{e}[\mathrm{~N} \mathrm{II}](\mathrm{K})$ & $16,500 \pm 300$ & $15,800 \pm 200$ \\
$\left.\mathrm{n}_{e} \mathrm{C} \mathrm{III}\right]\left(\mathrm{cm}^{-3}\right)$ & 36,500 & 36,500 \\
$\mathrm{n}_{e}[\mathrm{~S} \mathrm{II}]\left(\mathrm{cm}^{-3}\right)$ & 299 & 9 \\
& & \\
$\mathrm{O}^{+} / \mathrm{H}^{+}\left(10^{-5}\right)$ & $0.19 \pm 0.01^{a}$ & $0.20 \pm 0.01$ \\
$\mathrm{O}^{+2} / \mathrm{H}^{+}\left(10^{-5}\right)$ & $2.21 \pm 0.07$ & $2.53 \pm 0.08$ \\
$12+\log (\mathrm{O} / \mathrm{H})$ & $7.38 \pm 0.01$ & $7.44 \pm 0.01$ \\
& & \\
$\mathrm{C}^{+2} / \mathrm{H}^{+}\left(10^{-5}\right)$ & $3.18 \pm 0.40$ & $4.42 \pm 0.60$ \\
$\mathrm{C}^{+3} / \mathrm{H}^{+}\left(10^{-5}\right)$ & $0.60 \pm 0.01$ & $0.88 \pm 0.02$ \\
$\mathrm{O}^{+2} / \mathrm{H}^{+}\left(10^{-5}\right)$ & $4.95 \pm 0.12$ & $7.53 \pm 0.20$ \\
$\mathrm{C}^{+2} / \mathrm{O}^{+2}$ & $0.64 \pm 0.05$ & $0.59 \pm 0.05$ \\
$\log (\mathrm{C} 3 \mathrm{C} 4)$ & 0.727 & 0.701 \\
$\log \mathrm{U}$ & -2.04 & -2.05 \\
$\mathrm{ICF}$ & $-0.16 \pm 0.04$ & $-0.20 \pm 0.080$ \\
$\log (\mathrm{C} / \mathrm{O})$ & & \\
& $0.89 \pm 0.03$ & $1.01 \pm 0.10$ \\
$\mathrm{~N}^{+} / \mathrm{H}^{+}\left(10^{-7}\right)$ & $-1.32 \pm 0.02$ & $-1.30 \pm 0.05$ \\
$\log (\mathrm{N} / \mathrm{O})$ & & \\
$\mathrm{S}^{+} / \mathrm{H}^{+}\left(10^{-7}\right)$ & $0.46 \pm 0.02$ & $0.45 \pm 0.03$ \\
$\mathrm{~S}^{+2} / \mathrm{H}^{+}\left(10^{-7}\right)$ & $2.69 \pm 0.10$ & $\cdots$ \\
$\log (\mathrm{S} / \mathrm{O})$ & $-1.41 \pm 0.04$ & $\cdots$ \\
& & \\
\hline
\end{tabular}

NoTE. - Ionic and total abundance calculations for SDSS and MMT observations of J082555. The ionization parameter is determined using $\log \left(\left[\mathrm{O}_{\mathrm{III}}\right] /[\mathrm{O} \mathrm{II}]\right)$ and the model photoionization diagnostic curves shown if Figures 4 and 5

${ }^{a} \mathrm{O}^{+} / \mathrm{H}^{+}$calculated from $[\mathrm{O}$ II] $\lambda \lambda 7320,7330$.

we expect our low metallicity sample to lie at low values of [N II] $\lambda 6584 / \mathrm{H} \alpha$. However, the distinct separation of detections and non-detections demonstrates that the UV O III] and C III] lines are most easily detected at very low abundances and become more difficult to observe at abundances typical of star-forming galaxies in the SDSS DR7 (gray dots).

Our requirement of large [O III $] \lambda 5007$ equivalent width helped select more high excitation targets, but the cut was not severe enough. The 5 non-detection targets in our sample have $54<\mathrm{EW}(5007)<390$, whereas our $\mathrm{C} / \mathrm{O}$ detections have significantly larger [O III $] \lambda 5007$ equivalent widths of $579<\mathrm{EW}(5007)<1275$. A similar, but less extreme division is found for $\mathrm{H} \beta$ equivalent width. In Figure 9(b) we plot the flux in O III] $\lambda 1666$ versus the [O III] $\lambda 5007$ equivalent width, demonstrating the the dichotomy in EW(5007) for detections (blue) and non-detections (red). The original estimates of the O III] $\lambda 1666$ flux based on the SDSS optical spectra (stars) are greater by a factor of $\sim 2$ compared to the actual measurements from the seven detections (filled circles). The vertical and horizontal dotted lines in Figure 9(b) divide up the $\mathrm{F}(\mathrm{OIII}] \lambda 1666)$ versus $\mathrm{EW}([\mathrm{OIII}] \lambda 5007)$ parameter space such that successful detections were produced by targets with predictions in the upper right hand quadrant. Thus, this diagram to useful to guide future sample selections.

We have argued that, given the currently available instruments/telescopes, simultaneously observing the $\mathrm{UV} \mathrm{O}^{+2}$ and $\mathrm{C}^{+2}$ CELs with HST/COS is the best method to measure $\mathrm{C} / \mathrm{O}$ abundances in nearby galaxies. Further, we now appreciate the importance of selecting the highest excitation targets that have $\mathrm{EW}([\mathrm{OIII}] \lambda 5007)$ measurements and predicted $\mathrm{F}(\mathrm{OIII}] \lambda 1666)$ values that place them in the upper right hand quadrant of Figure 9(b) Observing a larger sample of UV CELs constructed from these criteria is needed to confirm and delineate the C/O trend more clearly in nearby dwarf galaxies. 


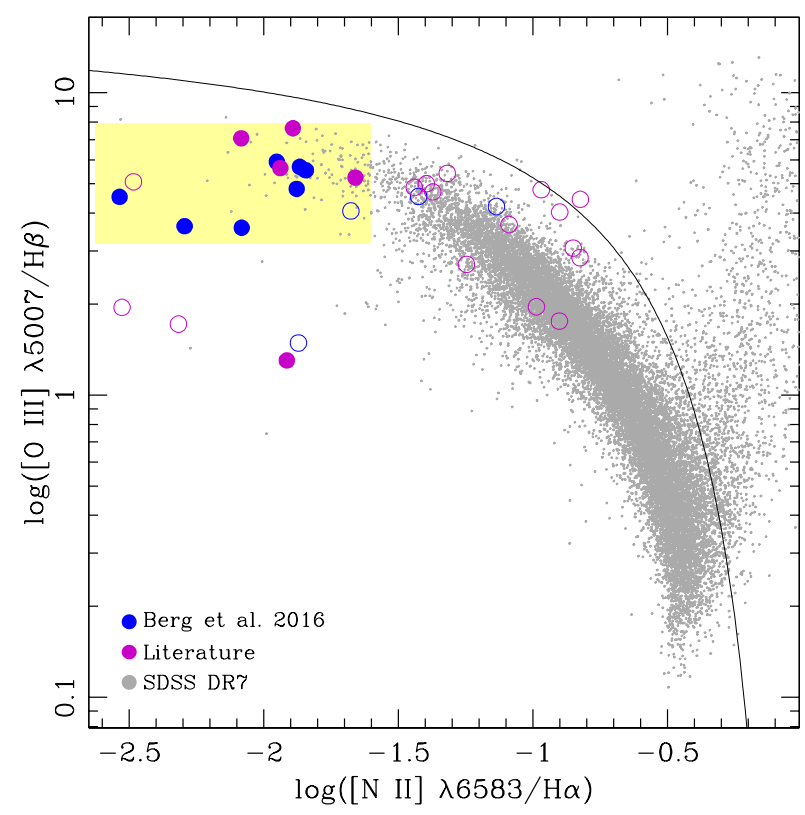

(a)

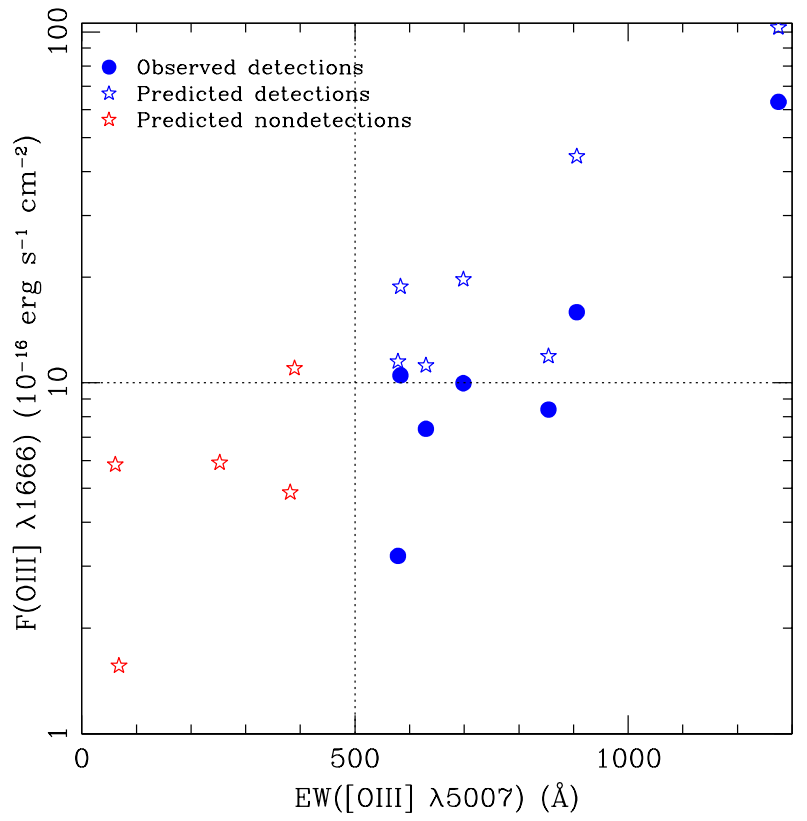

(b)

FIG. 9. - BPT diagram comparing the locations of targets with and without C/O detections. As before, blue symbols represent the $\mathrm{HST} / \mathrm{COS}$ observations presented here and purple symbols indicate line measurements take from other studies. $3 \sigma \mathrm{C} / \mathrm{O}$ detections are show as filled circles, while non-detections are shown as open circles. The yellow shaded box highlights BPT real-estate that has generally produced successful $\mathrm{C} / \mathrm{O}$ detections. (b) Flux in O III] $\lambda 1666$ versus the [O III] $\lambda 5007$ equivalent width plotted for predicted O III] $\lambda 1666$ fluxes based on the SDSS optical spectra (stars) and actual F O III] $\lambda 1666$ measurements (filled circles). C/O detections (3 $\sigma$ ) are blue and non-detections are red. This plot can be used to predict the success of future C/O detections (upper right had corner). 


\section{REFERENCES}

Abazajian, K., Adelman-McCarthy, J. K., Agüeros, M. A., \& others. 2005, AJ, 129, 1755

Abazajian, K. N., Adelman-McCarthy, J. K., Agüeros, M. A., et al. 2009, ApJS, 182, 543

Adelman-McCarthy, J. K., Agüeros, M. A., Allam, S. S., \& others. 2008, ApJS, 175, 297

Akerman, C. J., Carigi, L., Nissen, P. E., Pettini, M., \& Asplund, M. 2004, A\&A, 414, 931

Alam, S., Albareti, F. D., Allende Prieto, C., et al. 2015, ApJS, 219,12

Alexandroff, R., Strauss, M. A., Greene, J. E., et al. 2013, MNRAS, 435, 3306

Aoki, W., Beers, T. C., Christlieb, N., Norris, J. E., Ryan, S. G., \& Tsangarides, S. 2007, ApJ, 655, 492

Baldwin, J. A., Phillips, M. M., \& Terlevich, R. 1981, PASP, 93, 5

Bayliss, M. B., Rigby, J. R., Sharon, K., et al. 2014, ApJ, 790, 144

Beers, T. C. \& Christlieb, N. 2005, ARA\&A, 43, 531

Bensby, T. \& Feltzing, S. 2006, MNRAS, 367, 1181

Berg, D. A., Skillman, E. D., Croxall, K. V., et al. 2015, ApJ, 806,16

Berg, D. A., Skillman, E. D., Garnett, D. R., et al. 2013, ApJ, 775,128

Berg, D. A., Skillman, E. D., Marble, A. R., et al. 2012, ApJ, 754, 98

Bianchi, L., Conti, A., \& Shiao, B. 2014, Advances in Space Research, 53, 900

Binette, L., Matadamas, R., Hägele, G. F., et al. 2012, A\&A, 547, A29

Bohlin, R. C. 2010, AJ, 139, 1515

Bolton, A. S., Schlegel, D. J., Aubourg, É., \& others. 2012, AJ, 144, 144

Bresolin, F., Gieren, W., Kudritzki, R.-P., et al. 2009a, ApJ, 700, 309

Brinchmann, J., Charlot, S., White, S. D. M., Tremonti, C. Kauffmann, G., \& others. 2004, MNRAS, 351, 1151

Brinchmann, J., Kunth, D., \& Durret, F. 2008, A\&A, 485, 657

Brown, T. M., Tumlinson, J., Geha, M., Kirby, E. N., VandenBerg, D. A., Muñoz, R. R., Kalirai, J. S., Simon, J. D., Avila, R. J., Guhathakurta, P., Renzini, A., \& Ferguson, H. C. 2012, ApJ, 753, L21

Campbell, A., Terlevich, R., \& Melnick, J. 1986, MNRAS, 223, 811

Cardamone, C., Schawinski, K., Sarzi, M., et al. 2009, MNRAS, 399, 1191

Cardelli, J. A., Clayton, G. C., \& Mathis, J. S. 1989, ApJ, 345, 245

Carigi, L. 2000, Rev. Mexicana Astron. Astrofis., 36, 171

Carigi, L. \& Peimbert, M. 2011, Rev. Mexicana Astron. Astrofis. 47,139

Carigi, L., Peimbert, M., Esteban, C., \& García-Rojas, J. 2005, ApJ, 623, 213

Chiappini, C., Romano, D., \& Matteucci, F. 2003, MNRAS, 339, 63

Chieffi, A. \& Limongi, M. 2004, ApJ, 608, 405

Christensen, L., Laursen, P., Richard, J., et al. 2012, MNRAS, 427, 1973

Conroy, C. \& van Dokkum, P. G. 2012, ApJ, 760, 71

Cooke, R., Pettini, M., Steidel, C. C., Rudie, G. C., \& Nissen, P. E. 2011, MNRAS, 417, 1534

Dufour, R. J. 1984, PASP, 96, 787

Dufour, R. J., Shields, G. A., \& Talbot, Jr., R. J. 1982, ApJ, 252, 461

Eisenstein, D. J., Weinberg, D. H., Agol, E., et al. 2011, AJ, 142, 72

Ekta, B. \& Chengalur, J. N. 2010, MNRAS, 406, 1238

Erb, D. K. Pettini, M., Shapley, A. E., et al. 2010, ApJ, 719, 1168

Esteban, C., Bresolin, F., Peimbert, M., et al. 2009, ApJ, 700, 654

Esteban, C., García-Rojas, J., Carigi, L., et al. 2014, MNRAS, 443,624

Esteban, C., Peimbert, M., Torres-Peimbert, S., \& Rodríguez, M. 2002, ApJ, 581, 241

Fabbian, D., Nissen, P. E., Asplund, M., Pettini, M., \& Akerman, C. 2009 , A\&A, 500, 1143

Ferland, G. J., Porter, R. L., van Hoof, P. A. M., et al. 2013, Rev. Mexicana Astron. Astrofis., 49, 137

Fumagalli, M., da Silva, R. L., \& Krumholz, M. R. 2011, ApJ, 741, L26

García-Rojas, J. \& Esteban, C. 2007, ApJ, 670, 457

Garnett, D. R. 1990, ApJ, 363, 142

Garnett, D. R. 1992, AJ, 103, 1330

Garnett, D. R., Shields, G. A., Peimbert, M., et al. 1999, ApJ, 513,168

Garnett, D. R., Shields, G. A., Skillman, E. D., Sagan, S. P., \& Dufour, R. J. 1997, ApJ, 489, 63
Garnett, D. R., Skillman, E. D., Dufour, R. J., et al. 1995, ApJ 443,64

Gavilán, M., Buell, J. F., \& Mollá, M. 2005, A\&A, 432, 861

Gavilán, M., Mollá, M., \& Buell, J. F. 2006, A\&A, 450, 509

Green, J. C., Froning, C. S., Osterman, S., \& others. 2012, ApJ, 744,60

Gunn, J. E., Carr, M., Rockosi, C., \& others. 1998, AJ, 116, 3040

Guseva, N. G., Izotov, Y. I., Stasińska, G., Fricke, K. J., Henkel, C., \& Papaderos, P. 2011, A\&A, 529, A149

Guseva, N. G., Papaderos, P., Meyer, H. T., Izotov, Y. I., \& Fricke, K. J. 2009, A\&A, 505, 63

Gustafsson, B., Karlsson, T., Olsson, E., Edvardsson, B., \& Ryde, N. 1999, A\&A, 342, 426

Hainline, K. N., Shapley, A. E., Greene, J. E., \& Steidel, C. C. 2011, ApJ, 733, 31

Hainline, K. N., Shapley, A. E., Kornei, K. A., Pettini, M., Buckley-Geer, E., et al. 2009, ApJ, 701, 52

Henry, A., Scarlata, C., Martin, C. L., \& Erb, D. 2015, ApJ, 809 19

Henry, R. B. C., Edmunds, M. G., \& Köppen, J. 2000, ApJ, 541, 660

Hirschi, R. 2007, A\&A, 461, 571

Hirschi, R. \& et al. 2006, in Reviews in Modern Astronomy, Vol. 19, Reviews in Modern Astronomy, ed. S. Roeser, 101

Izotov, Y. I., Guseva, N. G., Fricke, K. J., \& Papaderos, P. 2009 A\&A, 503, 61

Izotov, Y. I. \& Thuan, T. X. 1999, ApJ, 511, 639

Izotov, Y. I., Thuan, T. X., \& Guseva, N. G. 2012, A\&A, 546 A122

James, B. L., Pettini, M., Christensen, L., et al. 2014, MNRAS, 440,1794

Jenkins, E. B. 2014, ArXiv e-prints

Jester, S., Schneider, D. P., Richards, G. T., et al. 2005, AJ, 130, 873

Kalirai, J. S., Anderson, J., Dotter, A., et al. 2013, ApJ, 763, 110

Kauffmann, G., Heckman, T. M., White, S. D. M., \& others. 2003, MNRAS, 341, 33

Kennicutt, Jr., R. C. 1998, ApJ, 498, 541

Kennicutt, Jr., R. C., Bresolin, F., \& Garnett, D. R. 2003, ApJ, 591,801

Kewley, L. J., Groves, B., Kauffmann, G., \& Heckman, T. 2006 MNRAS, 372, 961

Kniazev, A. Y., Pustilnik, S. A., Grebel, E. K., Lee, H., \& Pramskij, A. G. 2004, ApJS, 153, 429

Kobulnicky, H. A. \& Skillman, E. D. 1997, ApJ, 489, 636

Kobulnicky, H. A. \& Skillman, E. D. 1998, ApJ, 497, 601

Kroupa, P. 2001, MNRAS, 322, 231

Kroupa, P., Tout, C. A., \& Gilmore, G. 1993, MNRAS, 262, 545

Kurt, C. M., Dufour, R. J., Garnett, D. R., et al. 1999, ApJ, 518, 246

Lee, J. C., Gil de Paz, A., Tremonti, C., et al. 2009, ApJ, 706, 599

Leitherer, C., Schaerer, D., Goldader, J. D., et al. 1999, ApJS, 123,3

Limongi, M. \& Chieffi, A. 2003, ApJ, 592, 404

López-Sánchez, Á. R., Esteban, C., García-Rojas, J., Peimbert, M., \& Rodríguez, M. 2007, ApJ, 656, 168

Maeder, A. 1990, A\&AS, 84, 139

- 1992, A\&A, 264, 105

Maeder, A., Meynet, G., \& Chiappini, C. 2015, A\&A, 576, A56

Marassi, S., Chiaki, G., Schneider, R., et al. 2014, ApJ, 794, 100

Marigo, P., Bressan, A., \& Chiosi, C. 1996, A\&A, 313, 545

- 1998, A\&A, 331, 564

Mateo, M. L. 1998, ARA\&A, 36, 435

Mattsson, L. 2010, A\&A, 515, A68

Meynet, G., Ekström, S., \& Maeder, A. 2006, A\&A, 447, 623

Meynet, G., Hirschi, R., Ekstrom, S., Maeder, A., Georgy, C., Eggenberger, P., \& Chiappini, C. 2010, A\&A, 521, A30

Meynet, G. \& Maeder, A. 2002, A\&A, 390, 561

Meynet, G., Maeder, A., \& Hirschi, R. 2003, ArXiv Astrophysics e-prints

Mollá, M., Cavichia, O., Gavilán, M., \& Gibson, B. K. 2015, MNRAS, 451, 3693

Nava, A., Casebeer, D., Henry, R. B. C., \& Jevremovic, D. 2006 ApJ, 645, 1076

Nomoto, K., Kobayashi, C., \& Tominaga, N. 2013, ARA\&A, 51 457

Peimbert, A. 2003, ApJ, 584, 735

Peimbert, A. \& Peimbert, M. 2010, ApJ, 724, 791

Peimbert, A., Peimbert, M., \& Ruiz, M. T. 2005, ApJ, 634, 1056

Peimbert, M. 1967, ApJ, 150, 825

Peimbert, M., Pena, M., \& Torres-Peimbert, S. 1986, A\&A, 158 266

Pettini, M., Zych, B. J., Steidel, C. C., \& Chaffee, F. H. 2008,

MNRAS, 385, 2011

Pilyugin, L. S. \& Thuan, T. X. 2005, ApJ, 631, 231 
Portinari, L., Chiosi, C., \& Bressan, A. 1998, A\&A, 334, 505

Quider, A. M., Pettini, M., Shapley, A. E., \& Steidel, C. C. 2009 MNRAS, 398, 1263

Salpeter, E. E. 1955, ApJ, 121, 161

Salvadori, S., Skúladóttir, Á., \& Tolstoy, E. 2015, MNRAS, 454, 1320

Shapley, A. E., Steidel, C. C., Pettini, M., \& Adelberger, K. L. 2003, ApJ, 588, 65

Simón-Díaz, S. \& Stasińska, G. 2011, A\&A, 526, A48

Skillman, E. D. \& Kennicutt, Jr., R. C. 1993, ApJ, 411, 655

Spite, M., Caffau, E., Bonifacio, P., et al. 2013, A\&A, 552, A107

Spite, M., Cayrel, R., Plez, B., et al. 2005, A\&A, 430, 655

Stark, D. P., Richard, J., Siana, B., et al. 2014, MNRAS, 445, 3200

Stasińska, G. 1982, A\&AS, 48, 299

Terlevich, R. \& Melnick, J. 1985, MNRAS, 213, 841

Thuan, T. X., Izotov, Y. I., \& Lipovetsky, V. A. 1995, ApJ, 445 , 108
Thuan, T. X., Izotov, Y. I., \& Lipovetsky, V. A. 1996, ApJ, 463, 120

Tolstoy, E., Hill, V., \& Tosi, M. 2009, ARA\&A, 47, 371

Tominaga, N., Iwamoto, N., \& Nomoto, K. 2014, ApJ, 785, 98

Torres-Peimbert, S., Peimbert, M., \& Daltabuit, E. 1980, ApJ, 238,133

Tremonti, C. A., Heckman, T. M., Kauffmann, G., et al. 2004, ApJ, 613, 898

Tsujimoto, T. \& Bekki, K. 2011, A\&A, 530, A78

van den Hoek, L. B. \& Groenewegen, M. A. T. 1997, A\&AS, 123

van Zee, L., Salzer, J. J., \& Haynes, M. P. 1998, ApJ, 497, L1

Venn, K. A. 1995, ApJ, 449, 839

Wheeler, J. C., Sneden, C., \& Truran, Jr., J. W. 1989, ARA\&A, 27,279

York, D. G., Adelman, J., Anderson, Jr., J. E., et al. 2000, AJ, 120,1579 